Homology, Homotopy and Applications, vol.6(1), 2004, pp.59-85

\title{
LIE ALGEBRA COHOMOLOGY AND GENERATING FUNCTIONS
}

\author{
ALEXEI TOLPYGO \\ (communicated by Hvedri Inassaridze)
}

Abstract

Let $\mathfrak{g}$ be a simple Lie algebra, $V$ an irreducible $\mathfrak{g}$-module, $W$ the Weyl group and $\mathfrak{b}$ the Borel subalgebra of $\mathfrak{g}, \mathfrak{n}=[\mathfrak{b}, \mathfrak{b}]$, $\mathfrak{h}$ the Cartan subalgebra of $\mathfrak{g}$. The Borel-Weil-Bott theorem states that the dimension of $H^{i}(\mathfrak{n} ; V)$ is equal to the cardinality of the set of elements of length $i$ from $W$. Here a more detailed description of $H^{i}(\mathfrak{n} ; V)$ as an $\mathfrak{h}$-module is given in terms of generating functions.

Results of Leger and Luks and Williams who described $H^{i}(\mathfrak{n} ; \mathfrak{n})$ for $i \leqslant 2$ are generalized: $\operatorname{dim} H^{*}\left(\mathfrak{n} ; \Lambda^{*}(\mathfrak{n})\right)$ and $\operatorname{dim} H^{i}(\mathfrak{n} ; \mathfrak{n})$ for $i \leqslant 3$ are calculated and $\operatorname{dim} H^{i}(\mathfrak{n} ; \mathfrak{n})$ as function of $i$ and rank $\mathfrak{g}$ is described for the calssical series.

\section{Introduction}

The main field is $\mathbb{C}$ and all the algebras and modules considered are finite dimensional over $\mathbb{C}$. It is well known, that the standard cochain complex $C^{*}(\mathfrak{n} ; V)=$ $\oplus C^{k}(\mathfrak{n} ; V)$ is isomorphic to the space of linear maps from $\Lambda^{*} \mathfrak{n}$ into $V$; so

$$
C^{*}(\mathfrak{n} ; V) \cong\left(\Lambda^{*} \mathfrak{n}\right)^{\prime} \otimes V
$$

where prime denotes the dualization and the $\operatorname{sign}^{*}$ is reserved to denote the direct sum: for example, $H^{*}$ denotes $\underset{k}{\oplus} H^{k}, E_{1}^{*, *}=\oplus_{i, j} E_{1}^{i j}, E_{1}^{i, *}=\oplus E_{1}^{i, j}$, etc.

Let $\operatorname{diag}\left(a_{1}, \ldots, a_{n}\right)$ denote the matrix with the numbers $a_{i}$ on its main diagonal, the other elements being 0 .

Let $\mathfrak{g}$ be a (semi)simple Lie algebra, $V$ an irreducible $\mathfrak{g}$-module, $W$ the Weyl group of $\mathfrak{g}$ and $\mathfrak{b}$ its Borel subalgebra; $\mathfrak{n}=[\mathfrak{b}, \mathfrak{b}]$. According to the Borel-Weil-Bott (BWB) theorem [5] (whose different proofs are presented, with increasing clarity, e.g., in $[\mathbf{1 1}],[\mathbf{1}],[\mathbf{4}])$, we have

$$
\operatorname{dim} H^{i}(\mathfrak{n} ; V)=\left|W_{i}\right|
$$

Thanks are due to J. N. Bernstein and A. L. Onishchik for valuable advice and discussions of this paper, to D. A. Leites for editing and translating the manuscript and Stockholm University for financing the preprint of this text [20] and MPIM, Bonn, for the opportunity to make the paper avalable as preprint MPI-2003-17.

Received November 20, 2003; published on April 1, 2004.

2000 Mathematics Subject Classification: 17B56, 22E25

Key words and phrases: Lie algebra cohomology, Borel-Weil-Bott theorem.

(C) 2004, Alexei Tolpygo. Permission to copy for private use granted. 
where $|S|$ denotes the cardinality of $S$ and $W_{i}$ is the set of elements from $W$ of length $i$, see $[\mathbf{6}]$. We hope there will be no confusion of the cardinality of a set with the modulus of a polynomial introduced below.

In addition to $\mathfrak{g}$-modules, $\mathfrak{n}$ has a lot of natural modules which are not $\mathfrak{g}$-modules but which admit a $\mathfrak{b}$-module structure; and in this paper we will study such modules. The most interesting is the adjoint module $\mathfrak{n}$, and our main aim is to calculate cohomology with values in it. In particular, we will show (Theorem 7.6), that if $\mathfrak{g}=A_{r}$, i.e., $\mathfrak{g}=\mathfrak{s l}(r+1)$, then

$$
\operatorname{dim} H^{*}(\mathfrak{n} ; \mathfrak{n})=(r+1) ! \frac{r^{2}+9 r+2}{12} .
$$

Our methods can be generalized to embrace other $\mathfrak{b}$-modules.

Let $V$ be an arbitrary $\mathfrak{b}$-module; we will analyze the action of the Cartan subalgebra $\mathfrak{h}$ on the cochain complex $C^{*}(\mathfrak{n} ; V)$. This complex is a direct sum of its weight subspaces $C_{\nu}^{*}$ where $\nu \in \mathfrak{h}^{\prime}$. In $\S 2$ we will derive a formula for the generating function:

$$
F(t, x)=\sum_{k, \nu} \operatorname{dim} C_{\nu}^{k} \cdot t^{k} \cdot x^{\nu} .
$$

The guiding idea in what follows is that, first, the coboundary operator $d$ preserves each subcomplex $C_{\nu}^{*}=\oplus_{k} C_{\nu}^{k}$ and, second,

$$
\sum_{k}(-1)^{k} \operatorname{dim} H_{\nu}^{k}=\sum_{k}(-1)^{k} \operatorname{dim} C_{\nu}^{k} .
$$

Therefore,

$$
\operatorname{dim} H^{*} \geqslant \sum_{\nu}\left|\sum_{k}(-1)^{k} \operatorname{dim} C_{\nu}^{k}\right| .
$$

Inequality (0.3) coincides with the inequality (2.5) and we will investigate for which modules this inequality becomes an equality. The modules for which the equality is attained are called blue ones. ${ }^{1}$

In $\S 4$ we will show that any irreducible $\mathfrak{g}$-module $V$ is blue, and in $\S 6$ we prove the same for $V=\mathfrak{n}$, if $\mathfrak{g}$ is of type $A_{r}$.

If $V$ is a blue module, one can calculate its cohomology simply by counting the coefficients of the function $G(x)=F(-1, x)$. In $\S 7$ we will calculate in this way $\operatorname{dim} H^{*}(\mathfrak{n} ; \mathfrak{n})$ and $\operatorname{dim} H^{k}(\mathfrak{n} ; \mathfrak{n})$ for $k \leqslant 3$. In $\S 8$ we will establish some properties of $\operatorname{dim} H^{k}(\mathfrak{n} ; \mathfrak{n})$ regarded as a function of $k$ and rk $\mathfrak{g}$.

In $\S 9$ we will discuss the results and problems for $\mathfrak{g} \neq A_{r}$.

I do not know any other investigations concerning $\operatorname{dim} H^{*}(\mathfrak{n} ; V)$, where $V$ is not a $\mathfrak{g}$-module, except calculations of Leger and Luks [12] and Williams [21], where $H^{2}(\mathfrak{n} ; \mathfrak{n})$ and $H^{1}(\mathfrak{n} ; V)$ for $V$ equal to either $\mathfrak{n}$, or its dual, $\mathfrak{n}^{\prime}$, or $\mathfrak{g} / \mathfrak{n}$ are calculated.

\footnotetext{
${ }^{1}$ As opposed to yellow modules, the colors chosen to match the two colors of the banner of the independent Ukraine.
} 
Some results of this paper were announced in $[\mathbf{1 5}]$, see also [16]-[19]. They were delivered at the seminars of A. L. Onishchik-É. B. Vinberg and D. A. Leites in $1978 / 79$.

\section{$\S 1$. Generating Functions}

\section{1}

Let $x=\left(x_{1}, \ldots, x_{r}\right)$ be an $r$-tuple. For $\nu=\left(\nu_{1}, \ldots, \nu_{r}\right) \in \mathbb{R}^{r}$, we set $x^{\nu}=$ $x_{1}^{\nu_{1}} \ldots x_{r}^{\nu_{r}}$. In this paper, except for $\$ \S 8-9$, a polynomial is an expression $P(x)=$ $\sum_{\nu} a_{\nu} \cdot x^{\nu}$ with real or complex coefficients, where $\nu$ runs over some finite set of real vectors.

For a polynomial $P$, we define its support as $N_{P}=\left\{\nu \in \mathbb{R}^{r} \mid a_{\nu} \neq 0\right\}$ and its modulus as $|P(x)|=\sum_{\nu \in N_{p}}\left|a_{\nu}\right|$. We call $P$ a convex polynomial if $N_{P}$ is a set of vertices of a convex polyhedral.

If $P(t, x)$ is a polynomial in two groups of indeterminates and $t^{0}=\left(t_{1}^{0}, \ldots, t_{s}^{0}\right)$ a vector with numerical coordinates, we denote by $\left|P\left(t^{0}, x\right)\right|$ the modulus of the polynomial obtained from $P(t, x)$ by replacing $t$ with $t^{0}$.

1.2. Proposition. If $P=\sum a_{\nu} x^{\nu}$ and $Q=\sum b_{\nu} x^{\nu}$ are polynomials, then

(i) $|P \cdot Q| \leqslant|P| \cdot|Q|$;

(ii) For any monomial $Q=x^{\nu}$, we have $|P \cdot Q|=|P|$.

(iii) If $P$ is convex and $b_{\nu}$ are integers, then $|P \cdot Q| \geqslant|P|$.

Proof. Statements (i) and (ii) are obvious. Now let $P$ be convex and $\mu \in N_{P}$. Then there exists a linear form $\varphi \in\left(\mathbb{R}^{r}\right)^{\prime}$ such that $\varphi(\mu)>\varphi(\nu)$ for all $\nu \in N_{P}$, $\nu \neq \mu$. We can find $\lambda \in N_{Q}$ such that $\varphi(\lambda) \geqslant \varphi\left(\nu^{\prime}\right)$ for any $\nu^{\prime} \in N_{Q}$; then $\varphi(\lambda+\mu)>\varphi\left(\nu+\nu^{\prime}\right)$ and, therefore, $\lambda+\mu \neq \nu+\nu^{\prime}$ for any $\nu \in N_{P}, \nu^{\prime} \in M_{Q}$. So if $P \cdot Q=\sum c_{\nu} x^{\nu}$, then $\left|c_{\lambda+\mu}\right|=\left|a_{\mu} \cdot b_{\lambda}\right| \geqslant\left|a_{\mu}\right|$.

Thus, for any $\mu \in N_{P}$ we have found a vector $\lambda+\mu \in N_{P \cdot Q}$ such that $\left|c_{\lambda+\mu}\right| \geqslant$ $\left|a_{\mu}\right|$. Clearly, if $\mu \neq \mu_{1}$ then $\mu+\lambda \neq \mu_{1}+\lambda_{1}$. Hence,

$$
|P \cdot Q|=\sum_{\nu \in N_{P} \cdot Q}\left|c_{\nu}\right| \geqslant \sum_{\mu \in N_{P}}\left|c_{\lambda+\mu}\right| \geqslant \sum_{\mu \in N_{P}}\left|a_{\mu}\right|=|P|
$$

\section{3}

If a polynomial $Q$ is obtained from $P$ by an affine change of indeterminates, we will write $P \sim Q$.

Proposition. If $P \sim Q$, then $|P|=|Q|$.

Proof. The translation of the origin to the point $\nu$ corresponds to the multiplication of $P$ by $x^{\nu}$, so we may use Proposition 1.2(ii). Now, given a homogeneous change of indeteminates, let $\pi=\left(\pi_{1}, \ldots, \pi_{r}\right)$, where $\pi_{i}=\sum_{j} a_{i j} \nu_{j}$. Then we may denote:

$$
y_{i}=\prod_{j} x_{j}^{b_{i j}}, \text { where }\left(b_{i j}\right)=\left(a_{i j}\right)^{-1} .
$$


Clearly, $y^{\pi}=x^{\nu}$. This means that the change of indeteminates transforms a monomial $a_{\nu} x^{\nu}$ into some other monomial with the same coefficient $a_{\nu}$. Now our statement is obvious.

It is easy to see that the correspondence between the monomial $x^{\nu}$ and the $\delta$ function $\delta_{\nu}$ establishes an isomorphism of the polynomial ring and the group ring of $\mathbb{R}^{r}$. It will be more convenient for us to operate with the polynomial ring: to substitute numerical values of variables is more natural for polynomials.

\section{4}

Now let $\mathfrak{h}$ be an $r$-dimensional commutative Lie algebra and $V$ an $\mathfrak{h}$-module. Let $V$ be semisimple, i.e., let $V$ have a basis $v_{1}, \ldots, v_{m}$ such that $h \cdot v_{i}=\mu_{i}(h) \cdot v_{i}$ for any $h \in \mathfrak{h}$ and some $\mu=\left(\mu_{1}, \ldots, \mu_{m}\right) \in\left(\mathfrak{h}^{\prime}\right)^{m}$.

We will also assume that $\mathfrak{h}$ has a basis $h_{1}, \ldots, h_{r}$ such that all the numbers $\mu_{i}\left(h_{j}\right)$ are real. Then we may consider the generating function of the $\mathfrak{h}$-module $V$ :

$$
A_{V}(x)=\sum_{i=1}^{m} x^{\mu_{i}}
$$

This generating function coincides with the character of module $V$ in the terminology of [7]: if $V=\oplus V_{\nu}$ is the decomposition of $V$ into its weight subspaces, then, obviously,

$$
A_{V}(x)=\sum_{\nu} \operatorname{dim} V_{\nu} \cdot x^{\nu}=\operatorname{ch} V .
$$

If $V=\underset{k \in \mathbb{Z}}{\oplus} V^{k}$ is a $\mathbb{Z}$-graded module, we will consider the generating function

$$
A_{V}(t, x)=\sum_{k, \nu} \operatorname{dim} V_{\nu}^{k} \cdot t^{k} \cdot x^{\nu}
$$

where $t$ is one more (one-dimensional) indeterminate.

1.5. Proposition. Let $U$ and $V$ be two semisimple $\mathfrak{h}$-modules, $A_{U}(x)$ and $A_{V}(x)$ their generating functions (1.1). Then the $\mathfrak{h}$-modules $U \oplus V, U \otimes V, V^{\prime}$ and $\Lambda^{*} V=$ $\oplus \Lambda^{k} V$ are also semisimple and their generating functions are given by the formulas

$$
\begin{gathered}
A_{U \oplus V}=A_{U}+A_{V}, \\
A_{U \otimes V}=A_{U} \cdot A_{V}, \\
A_{V^{\prime}}(x)=A_{V}\left(x^{-1}\right)=\sum_{i} x^{-\mu_{i}}, \\
A_{\Lambda^{*} V}(t, x)=\prod_{i}\left(1+t \cdot x^{\mu_{i}}\right) .
\end{gathered}
$$

Proof is obvious. 


\section{§2. $\mathfrak{b}$-n-modules}

\section{1}

Now let $\mathfrak{n}$ be an arbitrary Lie algebra and $V$ any $\mathfrak{n}$-module. A pair $(D, A)$ is a derivation of $V$ compatible with $D$ if $D$ is a derivation of $\mathfrak{n}$ and $A: V \longrightarrow V$ is a linear transformation such that

$$
A(n \cdot v)=n \cdot A v+D n \cdot v \text { for any } n \in \mathfrak{n}, v \in V .
$$

Obviously such derivations form a Lie algebra, which we denote by $\mathfrak{d e r}(V)$. There is a projection: $(D, A) \mapsto D$ from $\mathfrak{d e r}(V)$ on $\mathfrak{d e r}(\mathfrak{n})$; its kernel consists of all pairs $(0, A)$, where $A: V \longrightarrow V$ commutes with the $\mathfrak{n}$-action on $V$. This projection gives us the action of $\mathfrak{d} \mathfrak{e r}(V)$ on $\mathfrak{n}$, and, therefore, we can define the action of $\mathfrak{d e r}(V)$ on the cochain complex $C^{*}(\mathfrak{n} ; V)$ by the standard formula

$$
((D, A) f)\left(n_{1}, \ldots, n_{k}\right)=A f\left(\left(n_{1}, \ldots, n_{k}\right)-\sum f\left(\left(n_{1}, \ldots, D n_{i}, \ldots, n_{k}\right) .\right.\right.
$$

The standard calculation shows that the coboundary operator $d: C^{k} \longrightarrow C^{k+1}$ commutes with this action of $\mathfrak{d e r}(V)$ on $C^{*}(\mathfrak{n} ; V)$. So this action induces an action of $\mathfrak{d e r}(V)$ on $H^{*}(\mathfrak{n} ; V)$.

\section{2}

Now, let $\mathfrak{b}$ be Lie algebra, $\mathfrak{n}$ - ideal in $\mathfrak{b}$, and let $V$ be a $\mathfrak{b}$-module (so $V$ is an $\mathfrak{n}$-module as well). For any $b \in \mathfrak{b}$ let $D_{b}$ be the restriction of ad $b$ onto $\mathfrak{n}$ and $A_{b}$ the action of $b$ on $V$. Then

$$
b \mapsto\left(D_{b}, A_{b}\right)
$$

is a homomorphism $\mathfrak{b} \longrightarrow \mathfrak{d e r}(V)$. So $\mathfrak{b}$ naturally acts on $C^{*}(\mathfrak{n} ; V)$ and on $H^{*}(\mathfrak{n} ; V)$. It is well known that every Lie algebra trivially acts on its (co)homology, so $\mathfrak{n}$ trivially acts on $H^{*}(\mathfrak{n} ; V)$, and we may regard the above $\mathfrak{b}$-action as the action of $\mathfrak{b} / \mathfrak{n}=\mathfrak{h}$ on $H^{*}(\mathfrak{n} ; V)$.

\section{3}

Henceforth we will assume that $\mathfrak{b}$ is a semidirect sum of its ideal $\mathfrak{n}$ and a commutative subalgebra $\mathfrak{h}$. Then any $\mathfrak{b}$-module is at the same time an $\mathfrak{h}$-module and an $\mathfrak{n}$-module. We will call $V$ a $\mathfrak{b}$-n-module if

(i) $V$ is a $\mathfrak{b}$-module and

(ii) $V$ has a basis of $\mathfrak{h}$-eigenvectors $v_{1}, . ., v_{m}$ with real weights in some fixed basis of $\mathfrak{h}$.

The weight of $v_{i}$ will be denoted by $\mu_{i} \in \mathfrak{h}^{\prime}$. We will assume that all the modules to be studied are $\mathfrak{b}$-n-modules; in particular, we will assume that the adjoint representation of $\mathfrak{h}$ on $\mathfrak{n}$ is semisimple; let $\lambda_{1}, \ldots, \lambda_{n} \in \mathfrak{h}^{\prime}$ be the weights of this representation.

2.4

Let $C^{*}=\underset{\nu}{\oplus} C_{\nu}^{*}=\underset{k, \nu}{\oplus} C_{\nu}^{k}$ be the decomposition of the graded $\mathfrak{h}$-module $C^{*}=$ $C^{*}(\mathfrak{n} ; V)$. Set $a_{k \nu}=\operatorname{dim} C_{\nu}^{k}$. 
Theorem. Let $F_{V}(t, x)=\sum_{k, \nu} a_{k \nu} \cdot t^{k} \cdot x^{\nu}$ be the generating function of $C^{*}(\mathfrak{n} ; V)$. Then

$$
F_{V}(t, x)=\prod_{i}\left(1+t \cdot x^{-\lambda_{i}}\right) \cdot A_{V}(x) .
$$

Proof. Since $C^{*}(\mathfrak{n} ; V)$ is isomorphic to $\left(\Lambda^{*} \mathfrak{n}\right)^{\prime} \otimes V$ as a graded $\mathfrak{h}$-module (formula $(0.3)$ ), our theorem follows from Proposition1.5.

\section{5}

Set $b_{\nu}=\sum_{0 \leqslant k \leqslant n}(-1)^{k} a_{k \nu}$ and $c_{k \nu}=a_{k \nu}-a_{k+1 \nu}-a_{k-1 \nu} ;$ let $G_{V}(x)=\sum b_{\nu} \cdot x^{\nu}$. Obviously, $G_{V}(x)=F_{V}(-1, x)$.

Theorem. In the above notations

$$
\begin{gathered}
\operatorname{dim} H^{*}(\mathfrak{n} ; V) \geqslant\left|G_{V}\right| ; \\
\operatorname{dim} H_{\nu}^{k} \geqslant c_{k \nu} ; \\
\operatorname{dim} H^{k} \geqslant \sum_{\nu} \max \left(0, c_{k \nu}\right) .
\end{gathered}
$$

Proof. Denote by $H_{\nu}^{k}$ is the cohomology of the complex

$$
\ldots \longrightarrow C_{\nu}^{k-1} \longrightarrow C_{\nu}^{k} \longrightarrow C_{\nu}^{k+1} \longrightarrow \ldots
$$

Clearly,

$$
\operatorname{dim} H_{\nu}^{k} \geqslant \operatorname{dim} C_{\nu}^{k}-\operatorname{dim} C_{\nu}^{k-1}-\operatorname{dim} C_{\nu}^{k+1}=c_{k \nu}
$$

and we obtain (2.6). It immediately implies (2.7).

To prove formula (2.5), let us apply $(0.2)$ to the complex $C_{\nu}^{*}$. We get

$$
\left|b_{\nu}\right|=\left|\sum_{k}(-1)^{k} \operatorname{dim} C_{\nu}^{k}\right|=\left|\sum_{k}(-1)^{k} \operatorname{dim} H_{\nu}^{k}\right| \leqslant \sum_{k} \operatorname{dim} H_{\nu}^{k}=\operatorname{dim} H_{\nu}^{*} .
$$

Hence, $\operatorname{dim} H^{*}=\sum_{\nu} \operatorname{dim} H_{\nu}^{*} \geqslant \sum\left|b_{\nu}\right|=|G|$.

2.6. Corollary. If $\lambda_{1} \neq 0, \ldots, \lambda_{n} \neq 0$ and $V$ is a $\mathfrak{b}$-n-module, then $H^{*}(\mathfrak{n} ; V) \neq 0$.

Proof. If $H^{*}(\mathfrak{n} ; V)=0$, then $|G|=0$ and so $G(x) \equiv 0$. Since $G(x)=F(-1, x)$, we see from (2.4) that some of the vectors $\lambda_{1}, \ldots, \lambda_{n}$ vanish.

The assumption on $V$ to be a $\mathfrak{b}$-n-module is essential. If $\mathfrak{b}$ is a two-dimensional non-commutative Lie algebra, $\mathfrak{n}=[\mathfrak{b}, \mathfrak{b}]$ is the one-dimensional Lie algebra and $V$ is one-dimensional non-trivial $\mathfrak{n}$-module, then $H^{*}(\mathfrak{n} ; V)=0$ even if $\lambda \neq 0$.

2.7. Corollary. Let $z^{0}=\left(z_{1}^{0}, \ldots, z_{s}^{0}\right)$ be a set of complex numbers, $\left|z_{i}^{0}\right| \leqslant 1$ for all $i$, and $y=\left(y_{1}, \ldots, y_{r-s}\right)$ some variables. Then $\operatorname{dim} H^{*}(\mathfrak{n} ; V) \geqslant\left|G\left(z^{0}, y\right)\right|$. 


\section{$\S 3$. The spectral sequence. The term $E_{1}^{i j}$}

\section{1}

If $V=\mathbb{C}$ is the trivial $\mathfrak{b}$-module, then $A_{V}(x)=1$. So $F_{\mathbb{C}}(t, x)=\prod_{i}\left(1+t \cdot x^{\lambda_{i}}\right)$, and we can write

$$
F_{V}(t, x)=F_{\mathbb{C}}(t, x) A_{V}(x) .
$$

Our purpose in this section is to set an analogous formula (3.5) for the generating functions of cohomology.

\section{2}

Let $V$ be a $\mathfrak{b}$-n-module. Assume that $V$ admits a filtration

$V=V_{m} \supset V_{m-1} \supset \ldots \supset V_{1} \supset V_{0}=\{0\}$ such that $\operatorname{dim} V_{i}=i$ and $\mathfrak{b} V_{i} \subset V_{i} ; \quad \mathfrak{n} V_{i} \subset V_{i-1}$.

If (3.2) is fulfilled, $V$ is said to be a nilmodule. One can easily see that if there exists an $h \in \mathfrak{h}$ such that $\lambda_{i}(h)>0$ for all $i$, then any such $\mathfrak{b}$-n-module $V$ is a nilmodule.

Denote: $C^{(i)}=\left\{\varphi \in C^{*} \mid \operatorname{Im} \varphi \subset V_{i}\right\}$. Then the sets $C^{(i)}$ forms a filtration of $C^{*}$, and this filtration generates a spectral sequence $[\mathbf{9}]$. Let us calculate its first term $E_{1}^{i j}$.

3.3. Theorem. $E_{1}^{i j} \cong H^{i}(\mathfrak{n})$ as linear spaces for all $j$ such that $0 \leqslant j \leqslant \operatorname{dim} V-1$.

Proof. The main rule for calculating $E_{1}$ is to replace the filtered complex with the graded one. In our case this means to substitute the graded module $V_{g r}$ in place of the filtered module $V$. So $E_{1}^{i j} \cong H^{i}\left(\mathfrak{n} ; V_{j+1} / V_{j}\right) \cong H^{i}(\mathfrak{n})$.

Usually, the differentials $d_{k}$ in the spectral sequence map $E_{k}^{i j}$ to $E_{k}^{i+1-k, j+k}$, and $\oplus E_{\infty}^{i-j, j}$ is the graded module associated with the filtered module $H^{i}$. But our $j$ method of filtration does not coincide with the standard one: here $d_{k}$ maps $E_{k}^{i j}$ to $E_{k}^{i+1, j+k}$, and the graded module associated with $H^{i}(\mathfrak{n} ; V)$ is $\underset{j=0}{\stackrel{m-1}{\oplus}} E_{\infty}^{i j}=E_{\infty}^{i *}$.

\subsection{The action of $\mathfrak{b}$ on the spectral sequence}

Theorem. The action of $\mathfrak{b}$ on $C^{*}$ induces an action of $\mathfrak{b}$ on $E_{k}^{i j}$ for all $i, j, k$. This action commutes with $d_{k}$ and its restriction on $\mathfrak{n}$ is trivial.

Proof. Substitution of $V_{g r}$ for $V$ means that we regard the elements of $V_{i-1}$ as "infinitesimals" as compared with the elements of $V_{i}$. (One can use the language of contracted representations [13], [2] to give the formal proof.) We know that $d$ commutes with the action of $\mathfrak{b}$. So it commutes "even more so" if we neglect infinitesimals, and $E_{1}^{*}$ admits a $\mathfrak{b}$-module structure. The second of the formulas (3.2) shows that $\mathfrak{n}$ acts trivially on $V_{g r}$ and, therefore, $\mathfrak{n}$ acts trivially on $E_{1}^{*}$ (hence, $\mathfrak{n}$ acts trivially on $\left.E_{k}^{*}\right)$. Thus we have proved the theorem for $k=1$. It remains to regard the infinitesimals up to the $k$-th order as negligible ones which gives the proof for any $k$. 


\subsection{Main result of this section}

Let $h_{k \nu}=\operatorname{dim} H_{\nu}^{k}(\mathfrak{n})$ and let

$$
P(t, x)=\sum_{k, \nu} h_{k \nu} \cdot t^{k} \cdot x^{\nu}
$$

be the generating function for the graded $\mathfrak{h}$-module $H^{*}(\mathfrak{n})$.

Theorem. Let $V$ be $a \mathfrak{b}$-n-nilmodule and $V_{i}$ the terms of the corresponding filtration. Then the generating function $D(t, s, x)$ of the graded $\mathfrak{b}$-module $E_{1}^{*}=\oplus E_{1 \nu}^{i j}$ is given by the formula

$$
D_{V}(t, s, x)=P(t, x) \cdot \sum_{j} s^{j} \cdot x^{\mu_{j}},
$$

where $\mu_{j}$ is the weight of $V_{j+1} / V_{j}$.

Proof. Obviously, $D(t, s, x)=\sum_{j} s^{j} \cdot D_{j}(t, x)$, where $D_{j}$ is the generating function for $E_{1}^{* j}$. Now let $u_{1}$ be the weight vector from $E_{1}^{* j}$ and $u_{0}$ its representative in $E_{0}^{* j}=\left(\Lambda^{*} \mathfrak{n}\right)^{\prime} \otimes\left(V_{j+1} / V_{j}\right)$.

We see that the weights of $u_{1}$ and $u_{0}$ coincide, and are equal to $\lambda=\mu_{j}$, where $\lambda$ is the weight of some cohomology class from $H^{*}(\mathfrak{n})$. So, by multiplying $P(t, x)$ by $x^{\mu_{j}}$, we obtain $D_{j}(t, x)$. This implies (3.4).

3.6. Corollary. $E_{1}^{*}$ is isomorphic to $H^{*}(\mathfrak{n}) \otimes V$ as $\mathfrak{h}$-modules.

Proof follows from the fact that the generating functions of these two $\mathfrak{h}$-modules coincide.

\section{7}

The function $D(t, s, x)$ is not invariant since one can consider filtration of $V$ with respect to different $s$ 's. So hereafter we will suppress the parameter $s$ and use the function $Q(t, x)=D(t, 1, x)$. Obviously,

$$
Q_{V}(t, x)=P(t, x) \cdot A_{V}(x) .
$$

So $Q$ only depends on the algebra $\mathfrak{b}$ and module $V$ over it.

3.8. Proposition. $Q_{V}(-1, x)=G_{V}(x)$.

Proof. Applying formula $(2.8)$ to the complex $C^{*}(\mathfrak{n})$ we see that $P(-1, x)=$ $F_{\mathbb{C}}(-1, x)$. Now it remains to use the definition of $G(x)$ and formulas (3.1), (3.5).

Therefore, hereafter we will use the function $Q_{V}$ instead of $F_{V}$.

\section{9}

We will also consider the function $R(x)=Q(1, x)$. Clearly, $|R(x)|=|Q(t, x)|=$ $\operatorname{dim} E_{1}^{*}$. So we see that

$$
\left|G_{V}(x)\right| \leqslant \operatorname{dim} H^{*}(\mathfrak{n} ; V) \leqslant\left|R_{V}(x)\right| .
$$

Let $r_{\nu}=\operatorname{dim} E_{1 \nu}^{*}$. Then $R(x)=\sum r_{\nu} \cdot x^{\nu}$. We will call $r_{\nu}=r_{\nu}(V)$ the $\nu$-multiplicity of the module $V$, and $r(V)=\max _{\nu} r_{\nu}(V)$ the multiplicity of $V$. 


\subsection{0}

Recall [4] that for the nilradical $\mathfrak{n}$ of $\mathfrak{b}$, there exists a free resolution of the trivial $\mathfrak{b}$-module such that $Q_{V}(t, x)$ is the generating function for the corresponding cochain complex for any $V$. We will only use a much simpler, trivial fact that $\operatorname{dim} H_{\nu}^{k} \leqslant \operatorname{dim} E_{1 \nu}^{k *}$; in particular, if $E_{1 \nu}^{k *}=0$, then $H_{\nu}^{k}=0$.

\section{$\S 4$. The Blue Modules and the Borel-Weil-Bott Theorem}

\section{1}

Now we will study the following question: when the inequality (2.5) becomes equality? Let us give an appropriate definition.

A $\mathfrak{b}$-n-module $V$ is a $\nu$-blue module if

$$
\operatorname{dim} H_{\nu}^{*}=\left|b_{\nu}\right|
$$

and $V$ is a blue module if it is $\nu$-blue for all the $\nu$.

So $V$ is a blue module if and only if $\operatorname{dim} H^{*}(\mathfrak{n} ; V)=\left|G_{V}\right|$.

4.2. Proposition. Let $V$ be $a \mathfrak{b}-\mathfrak{n}$-module and $\nu \in \mathfrak{h}^{\prime}$ a weight of $V$. The module $V$ is $\nu$-blue if and only if $H_{\nu}^{k}=0$ for all even $k$ or for all odd $k$.

Proof. Since $b_{\nu}=\sum_{k}(-1)^{k} \operatorname{dim} H_{\nu}^{k}$, then $V$ is $\nu$-blue if and only if

$$
\sum_{k} \operatorname{dim} H_{\nu}^{k}=\left|\sum_{k}(-1)^{k} \operatorname{dim} H_{\nu}^{k}\right| .
$$

So all the nonzero terms in the right-hand side must have the same sign.

4.3. Proposition. In the above notations any of the following conditions is sufficient for $V$ to be $\nu$-blue:

(i) $C_{\nu}^{k} \neq 0$ for no more than one $k$.

(ii) $H_{\nu}^{k} \neq 0$ for no more than one $k$.

(iii) $\operatorname{dim} C_{\nu}^{*} \leqslant 1$.

(iv) $r_{\nu} \leqslant 1$.

(v) $\operatorname{dim} H_{\nu}^{*} \leqslant 1$.

Proof. This is an easy corollary of Proposition 4.2 since any of the conditions (i)-(v) is stronger than the condition of Proposition 4.2.

\section{4}

Hereafter we will consider the situation discussed in Introduction: $\mathfrak{b}$ is the Borel subalgebra of some semisimple Lie algebra $\mathfrak{g}, \mathfrak{h}$ and $\mathfrak{n}$ are the Cartan subalgebra and the nilradical of $\mathfrak{b}$, respectively. Let $R_{+}=\left\{\alpha_{i}\right\}_{i \in I}$ be the set of positive roots of $\mathfrak{g}, R_{-}=-R_{+}, \rho=\frac{1}{2} \sum_{i \in I} \alpha_{i}$ and $W$ the Weyl group of $\mathfrak{g}$.

Clearly, if $U$ is a $\mathfrak{g}$-module, then $U$ is a $\mathfrak{b}$-n-nilmodule. So we can apply all the previous results to $U$. The weights of $U$ will be denoted by $\beta_{j}$ for $j=1, \ldots, m$.

Theorem. If $U$ is an irreducible $\mathfrak{g}$-module, then $U$ is a blue $\mathfrak{b}-\mathfrak{n}$-module.

Proof will be given in sec. 4.5-4.8. (One can prove that any finite dimensional $\mathfrak{g}$-module is blue.) 


\section{5}

We see that

$$
G_{V}(x)=\prod_{i \in I}\left(1-x^{-\alpha_{i}}\right) \cdot \sum_{j=1}^{m} x^{\beta_{j}} .
$$

Let us change the variables by setting $\pi=2 \nu-\rho$. Then $G_{V}(x)$ transforms into an equivalent function $\tilde{G}_{V}$, and, clearly,

$$
\tilde{G}_{V}(x)=\prod_{i \in I}\left(x^{\alpha_{i}}-x^{-\alpha_{i}}\right) \cdot \sum_{j=1}^{m} x^{2 \beta_{j}} .
$$

In what follows we will operate in these new variables.

4.6. Lemma. If $U=\mathbb{C}$ is the trivial $\mathfrak{g}$-module, then

$$
G_{\mathbb{C}}(x)=\sum_{w \in W}(-1)^{l(w)} \cdot x^{w \rho},
$$

where $l(w)$ is the length of $w \in W$, see $[\boldsymbol{6}]$. Moreover, $\left|G_{\mathbb{C}}\right|=|W|$.

Proof. One sees that the Weyl formula [7]

$$
\prod_{i \in I}\left(x^{\alpha_{i}}-x^{-\alpha_{i}}\right)=\sum_{w \in W}(-1)^{l(w)} \cdot x^{w \rho}
$$

coincides with (4.4). To prove the second statement, we must show that $w_{1} \rho \neq w_{2} \rho$ if $w_{1} \neq w_{2}$, because in this case there are $|W|$ different monomials in the right hand side of (4.4). But this follows from the well known fact that $\rho$ lies strictly inside a Weyl chamber.

4.7. Lemma. $G_{V} \geqslant|W|$ for any $\mathfrak{b}-\mathfrak{n}$-module.

Proof. Clearly, $|w \rho|=|\rho|$ for any $w$. So, by Lemma 4.6 the set $N_{G_{\mathbb{C}}}$ lies on the sphere of radius $|\rho|$ and, therefore, $G_{\mathbb{C}}$ is a convex polynomial. It remains to apply Proposition 1.2 (iii).

\section{8}

Now, by the BWB theorem, if $U$ is an irreducible $\mathfrak{g}$-module, then $\operatorname{dim} H^{*}(\mathfrak{n} ; U)=$ $|W|$. With Lemma 4.7, we have:

$$
|W| \leqslant G_{U} \leqslant \operatorname{dim} H^{*}(\mathfrak{n} ; U)=|W|
$$

which implies that all the inequalities are equalities. So $U$ is blue, and the proof of Theorem 4.4 is completed.

4.9. Corollary. $\operatorname{dim} H^{*}(\mathfrak{n} ; V) \geqslant|W|$ for any $\mathfrak{b}-\mathfrak{n}$-module $V$.

Proof follows from Lemma 4.7. 
4.10

Now we can give a convenient formula for the introduced in sec. 3.5 function $P(t, x)$. It follows from formula (4.4) and the fact that $\mathbb{C}$ is a blue module that for a function $m(w)$ we have

$$
P(t, x)=\sum_{w \in W} x^{w \rho} \cdot t^{m(w)} .
$$

Proposition. $m(w)$ in formula (4.7) coincides with $l(w)$.

Proof. It is well known [6] that the length of $w$ coincides with card $\left(w R_{+} \cap R_{-}\right)$. So let $l=l(w)$ and let $w\left\{\alpha_{1}, \ldots, \alpha_{n}\right\}=\left\{-\alpha_{1},-\alpha_{2}, \ldots,-\alpha_{l}, \alpha_{l+1}, \ldots, \alpha_{n}\right\}$. Then

$$
w \rho=\frac{1}{2}\left(-\alpha_{1}-\ldots-\alpha_{l}+\alpha_{l+1}+\ldots+\alpha_{n}\right) .
$$

Let us open the brackets in the left hand side of (4.5). We can get the monomial $x^{w \rho}$ only if we take each second summand from the first $l$ factors, and each first summand from all the other factors since the degree of any other monomial is less than that corresponding to $w \rho$ with respect to the Weyl chamber containing $w(\rho)$. Now, consider the corresponding to $G$ function $\tilde{F}_{\mathbb{C}}(t, x)=\prod\left(x^{\alpha_{i}}+t x^{-\alpha_{i}}\right)$; similarly, if we want to get the term $x^{w \rho} t^{m}$, we must take each second summand from the first $l$ factors. Thus, we get the term $x^{w \rho} t^{l}$.

Finally, we obtain:

$$
P(t, x)=\sum_{w} t^{l(w)} x^{w \rho}
$$

4.11. Theorem. Let $U$ be an irreducible $\mathfrak{g}$-module, $\nu$ an arbitrary weight. Then

(i) $\operatorname{dim} H_{\nu}^{*}(\mathfrak{n} ; U) \leqslant 1$.

(ii) $H_{\nu}^{*}(\mathfrak{n} ; U) \neq 0 \Longleftrightarrow \operatorname{dim} C_{\nu}^{*}(\mathfrak{n} ; U)=1$. So $\operatorname{dim} C_{\nu}^{*}(\mathfrak{n} ; U)$ is either even or is equal to 1 for any $\nu$.

(iii) Inequality (2.7) is an equality.

(iv) Let $\lambda_{+}$be the highest weight of $U$, and $\sigma=\rho+\lambda_{+}$. Then the generating function $P_{U}(t, x):=\sum_{k, \nu} \operatorname{dim} H_{\nu}^{k}(\mathfrak{n} ; U) \cdot t^{k} \cdot x^{\nu}$ is given by the formula

$$
P_{U}(t, x)=\sum_{w \in W} x^{w \sigma} \cdot t^{l(w)} .
$$

Proof is an easy consequence of the proof of Theorem 4.4 and Proposition 4.10.

\subsection{2}

It might be useful sometimes to have an explicit formula for $P(t, x)$ when $\mathfrak{g}$ is any classical simple Lie algebra. Taking $W$ and $\rho$ from $[6]$ we obtain Table 1.

If $\mathfrak{g}=A_{r}$, we see that $l(w)$ is equal to the number of inversions in the lower row of the permutation $w=\left(\begin{array}{cccc}0 & 1 & \ldots & r \\ i_{0} & i_{1} & \ldots & i_{r}\end{array}\right)$. 


\subsection{3}

Now we can find a convenient formula for the $\nu$-multiplicity on any $\mathfrak{b}$-n-module $V$. Let

$$
M_{\nu}=\left\{(w, j) \mid w \in W, j=1, \ldots, m, \quad w \rho+\beta_{j}=\nu\right\} .
$$

Let $L$ be the projection of $M_{\nu}$ on $\{1, \ldots, m\}$.

Proposition. $r_{\nu}=\operatorname{card} M_{\nu}=\operatorname{card} L_{\nu}$.

Proof. The first equality immediately follows from formulas (3.5) and (4.3) because $R(x)=Q(1, x)$. Now if $w_{1} \rho+\beta_{j_{1}}=w_{2} \rho+\beta_{j_{2}}$ and $w_{1} \neq w_{2}$, then, as we have seen, $w_{1} \rho \neq w_{2} \rho$ and so $j_{1} \neq j_{2}$. This gives the second equality.

Equivalently, we may describe $r_{\nu}$ as

$$
r_{\nu}=\operatorname{card}\left\{\mu \in N_{A_{V}} \mid \nu-\mu=w \rho \text { for some } w\right\} .
$$

\section{$\S 5$. Subquotients}

\section{1}

We will say that a module $V$ is a subquotient of $U$ if $V$ is isomorphic to a quotient module of a submodule of $U$ (in particular, if $V$ is a submodule or quotient module of $U$ ). We clearly see that if $V$ is any $\mathfrak{b}$-module in a $\mathfrak{b}$-subquotient of some $\mathfrak{g}$-module, then $V$ is a $\mathfrak{b}$-n-nilmodule.

Hereafter we will assume that all the modules are $\mathfrak{b}$-subquotients of a (usually irreducible) $\mathfrak{g}$-module. Let $U$ be a $\mathfrak{g}$-module, $V$ its subquotient; then we may regard $C^{*}(\mathfrak{g} ; U)$ as a $\mathfrak{g}$-module, and $C^{*}(\mathfrak{n} ; \tilde{U}), E_{1}^{*}(\mathfrak{n} ; \tilde{U}), H^{*}(\mathfrak{n} ; \tilde{U})$, where $\tilde{U}=U$ or $V$, are its subquotients.

\section{2}

In what follows the letter $U$ will always denote some $\mathfrak{g}$-module and $V$ its $\mathfrak{b}$ subquotient. Let $V_{0} \supset V_{1}$ be $\mathfrak{b}$-submodules of $U$ such that $V=V_{0} / V_{1}$. In $U$, we may find $\mathfrak{h}$-submodules $V_{2}$ and $V_{3}$, complementary to $V_{1}$ in $V_{0}$ and to $V_{0}$ in $U$, respectively. Evidently, $A_{V}(x)=A_{V_{2}}(x)$ and $V \cong V_{2}$, as h-modules; we will identify these modules, if necessary. We see also that the $V_{i}$ are $\mathfrak{b}$-subquotients of $U$ and $U \cong V_{1}+V_{2}+V_{3}$ as $\mathfrak{h}$-modules; so

$$
A_{U}(x)=A_{V_{1}}(x)+A_{V_{2}}(x)+A_{V_{3}}(x) .
$$

The number $\operatorname{cr}_{\nu}(V, U)=r_{\nu}(U)-r_{\nu}(V)$ is called the $\nu$-comultiplicity of $V$ with respect to $U$. Formula (5.1) implies that

$$
E_{1 \nu}^{*}(\mathfrak{n} ; U) \cong E_{1 \nu}^{*}\left(\mathfrak{n} ; V_{1}\right)+E_{1 \nu}^{*}\left(\mathfrak{n} ; V_{2}\right)+E_{1 \nu}^{*}\left(\mathfrak{n} ; V_{3}\right),
$$

so

$$
\operatorname{cr}_{\nu}(V, U)=r_{\nu}\left(V_{1}\right)+r_{\nu}\left(V_{3}\right) .
$$

5.3. Proposition. Let $U$ be an irreducible $\mathfrak{g}$-module, $V$ its $\mathfrak{b}$-subquotient and $\nu$ some weight such that $r_{\nu}(U) \neq 1$. Then

(i) $\operatorname{dim} H_{\nu}^{*}(\mathfrak{n} ; V) \leqslant r_{\nu}(V)$;

(ii) $\operatorname{dim} H_{\nu}^{*}(\mathfrak{n} ; V) \leqslant c r_{\nu}(V, U)$. 
Proof. (i) is clear, because

$$
\operatorname{dim} H_{\nu}^{*}(\mathfrak{n} ; V) \leqslant \operatorname{dim} E_{1 \nu}^{*}(\mathfrak{n} ; V)=r_{\nu}(V) .
$$

In particular, this means that $\operatorname{dim} H_{\nu}^{*}\left(\mathfrak{n} ; V_{i}\right) \leqslant r_{\nu}\left(V_{i}\right)$. Now, consider the exact sequence $0 \longrightarrow V_{1} \longrightarrow V_{0} \longrightarrow V \longrightarrow 0$. The corresponding exact cohomology sequence shows that

$$
\operatorname{dim} H_{\nu}^{*}(\mathfrak{n} ; V) \leqslant \operatorname{dim} H_{\nu}^{*}\left(\mathfrak{n} ; V_{1}\right)+\operatorname{dim} H_{\nu}^{*}\left(\mathfrak{n} ; V_{0}\right) .
$$

But the exact sequence $0 \longrightarrow V_{0} \longrightarrow U \longrightarrow V_{3} \longrightarrow 0$ shows that $H_{\nu}^{*}\left(\mathfrak{n} ; V_{0}\right) \cong$ $H_{\nu}^{*}\left(\mathfrak{n} ; V_{3}\right)$, since $H_{\nu}^{*}(\mathfrak{n} ; U)=0$ by Theorem 4.11 (ii). So $\operatorname{dim} H_{\nu}^{*}\left(\mathfrak{n} ; V_{0}\right) \leqslant r_{\nu}\left(V_{3}\right)$ and $\operatorname{dim} H_{\nu}^{*}\left(\mathfrak{n} ; V_{1}\right) \leqslant r_{\nu}\left(V_{1}\right)$. Now it only remains to apply formulas (5.3) and (5.2).

If $r_{\nu}(U)=1$, then (ii) fails but we clearly see that in this case $\operatorname{dim} H_{\nu}^{*}(\mathfrak{n} ; V)=$ $r_{\nu}(V)$ for any $V$.

5.4. Proposition. Let $U, V$, and $\nu$ be as in Proposition 5.3.

(i) If $r_{\nu}(V) \cdot \operatorname{cr}(V, U)=0$, then $\operatorname{dim} H_{\nu}^{*}(\mathfrak{n} ; V)=0$.

(ii) If $r_{\nu}(V)$ or $\operatorname{cr}(V, U)$ is equal to 1 , then $\operatorname{dim} H_{\nu}^{*}(\mathfrak{n} ; V)=1$.

(iii) $V$ is $\nu$-blue in all the cases mentioned in (i), (ii).

Proof. Since $r_{\nu}(U)$ is even, $r_{\nu}(V)$ and $\operatorname{cr}(V, U)$ are either simultaneously even or simultaneously odd and the same is true for $r_{\nu}(V)$ and $\operatorname{dim} H_{\nu}^{*}(\mathfrak{n} ; V)$.

So Proposition 5.3 implies (i) and (ii). Now (iii) is a consequence of Proposition $4.3(\mathrm{v})$.

5.5. Proposition. If $U$ is an irreducible $\mathfrak{g}$-module and $r_{\nu}(U) \leqslant 2$, then any $\mathfrak{b}$ submodule of $U$ is $\nu$-blue. In particular, if $r(U) \leqslant 2$, then any $\mathfrak{b}$-submodule of $U$ is blue.

Proof. If $r_{\nu}(U)=1$, we have to apply Proposition 4.3 (iv), otherwise we can apply Proposition 5.4 (iii), because $r_{\nu}(V) \leqslant 1$ or $c r_{\nu}(V, U) \leqslant 1$.

We see that the proposition is true if $r_{\nu}(U) \leqslant 3$, but such generalization is inessential, because we know that $\operatorname{dim} E_{1 \nu}^{*}(\mathfrak{n} ; U)$ is either even or is equal to 1 .

5.6. Proposition. Let $U$ be an irreducible $\mathfrak{g}$-module, $V$ its $\mathfrak{b}$-submodule and $\tilde{V}=$ $U / V$. Then $V$ is $\nu$-blue $\Longleftrightarrow \tilde{V}$ is $\nu$-blue for any $\nu$.

Proof. Let $G_{U}(x)=\sum b_{\nu} x^{\nu}, G_{V}(x)=\sum b_{\nu}^{\prime} x^{\nu}$ and $G_{\tilde{V}}=\sum b_{\nu}^{\prime \prime} x^{\nu}$ be the generating functions of the corresponding modules. As $A_{U}(x)=A_{V}(x)+A_{\tilde{V}}(x)$, we easily see that $G(x)=G_{V}(x)+G_{\tilde{V}}(x)$. So $b_{\nu}=b_{\nu}^{\prime}+b_{\nu}^{\prime \prime}$. Now we have to study two cases:

(1) If $b_{\nu} \neq 0$, then $\operatorname{dim} C_{\nu}^{*}(\mathfrak{n} ; U)=1$ (Theorem 4.11 (ii)), and both $V$ and $\tilde{V}$ are $\nu$-blue by Proposition 4.3 (ii).

(2) If $b_{\nu}=0$, then $b_{\nu}^{\prime}=\left|b_{\nu}^{\prime \prime}\right|$. But in this case $H_{\nu}^{*}(\mathfrak{n}, U)=0$, and the exact sequence $0 \longrightarrow V \longrightarrow U \longrightarrow \tilde{V} \longrightarrow 0$ shows that $H_{\nu}^{*}(\mathfrak{n} ; V) \cong H_{\nu}^{*}(\mathfrak{n} ; \tilde{V})$. So $b_{\nu}^{\prime}=\operatorname{dim} H_{\nu}^{*}(\mathfrak{n} ; V)$ if and only if $b_{\nu}^{\prime \prime}=\operatorname{dim} H_{\nu}^{*}(\mathfrak{n}, \tilde{V})$.

5.7. Proposition. Let $U$ be an irreducible $\mathfrak{g}$-module, $\nu$ one of its weights, $V$ and $V_{i}, i=1,2,3$, as in sec. 5.2. Suppose that $V$ is $\nu$-blue and $\operatorname{dim} H_{\nu}^{*}(\mathfrak{n} ; V) \geqslant$ $\operatorname{dim} H_{\nu}^{*}\left(\mathfrak{n} ; V_{1}\right)+\operatorname{dim} H_{\nu}^{*}\left(\mathfrak{n} ; V_{3}\right)$. Then both $V_{1}$ and $V_{3}$ are $\nu$-blue. 
Proof. Let $G_{U}, G_{V_{1}}, G_{V}$, and $G_{V_{3}}$ be the generating functions for $U, V_{1}, V \cong V_{2}$ and $V_{3}$ respectively. Then, as earlier, $b_{\nu}=b_{\nu}^{\prime}+b_{\nu}^{\prime \prime}+b_{\nu}^{\prime \prime \prime}=b_{\nu}^{\prime}+b_{\nu}^{\prime \prime \prime} \pm \operatorname{dim} H_{\nu}^{*}(\mathfrak{n} ; V)$. As the case $b_{\nu} \neq 0$ is trivial, we may suppose $b_{\nu}=0$, so

$$
\operatorname{dim} H_{\nu}^{*}(\mathfrak{n} ; V)=\left|b_{\nu}^{\prime}+b_{\nu}^{\prime \prime \prime}\right| \leqslant\left|b_{\nu}^{\prime}\right|+\left|b_{\nu}^{\prime \prime \prime}\right| \leqslant \operatorname{dim} H_{\nu}^{*}\left(\mathfrak{n} ; V_{1}\right)+\operatorname{dim} H_{\nu}^{*}\left(\mathfrak{n} ; V_{3}\right) .
$$

Hence, all the inequalities are equalities and (4.1) holds for $V_{1}, V_{3}$.

5.8. Proposition. $A \mathfrak{b}$-n-module $V$ is blue if and only if so is $V^{\prime}$. Moreover, $r(V)=$ $r\left(V^{\prime}\right)$.

Proof. By formula (1.6) the generating functions corresponding to $V$ and $V^{\prime}$ are

$$
G_{V}(x)=P(-1, x) \cdot A_{V}(x) \text { and } G_{V^{\prime}}(x)=P(-1, x) \cdot A_{V}\left(x^{-1}\right) .
$$

So we can change variables $x \mapsto x^{-1}$ and write:

$$
\left|G_{V^{\prime}}(x)\right|=\left|P(-1, x) \cdot A_{V}\left(x^{-1}\right)\right|=\left|P\left(-1, x^{-1}\right) \cdot A_{V}(x)\right| .
$$

But $P(-1, x)=G_{\mathbb{C}}(x)=\prod\left(x^{\alpha_{i}}-x^{-\alpha_{i}}\right)$, so we easily see that $P\left(-1, x^{-1}\right)=$ $\pm P(-1, x)$. Therefore $G_{V^{\prime}}\left(x^{-1}\right)= \pm G_{V}(x)$ and so $\left|G_{V^{\prime}}\right|=\left|G_{V}\right|$. On the other hand, it is well known that $\left(H^{*}(\mathfrak{n} ; V)\right)^{\prime} \cong H^{*}\left(\mathfrak{n} ; V^{\prime}\right)$ so $\operatorname{dim} H^{*}(\mathfrak{n} ; V)=\operatorname{dim} H^{*}\left(\mathfrak{n} ; V^{\prime}\right)$. The rest is clear.

Problem. Is it true that if $V$ is $\nu$-blue, then $V^{\prime}$ is $\nu$-blue?

In the rest of the paper we will use the methods developed above to calculate certain cohomology.

\section{$\S 6$. The adjoint representation of $\mathfrak{n}$ is blue for $\mathfrak{g}=A_{r}$}

This section is devoted to the proof of the following theorem:

6.1. Theorem. Let $\mathfrak{g}=A_{r}$ and $\mathfrak{n}$ the nilradical of its Borel subalgebra $\mathfrak{b}$. Then $\mathfrak{n}$, the adjoint $\mathfrak{n}$-module, is blue.

Proof will be given in sec. 6.2-6.9.

\section{2}

Recall that for $A_{r}=\mathfrak{s l}(r+1)$ we have: $\mathfrak{b}$ is the algebra of upper triangular matrices, $\mathfrak{h}$ the algebra of diagonal matrices and $\mathfrak{n}$ the algebra of strictly upper triangular matrices. We use the natural coordinates: if $h \in \mathfrak{h}$, then $h=\operatorname{diag}\left(h_{0}, \ldots, h_{r}\right)$ with $\sum h_{i}=0$; let $\nu=\left(\nu_{0}, \ldots, \nu_{r}\right)$ be the dual coordinates on $\mathfrak{h}^{\prime}$. So $\nu$ has $r+1$ coordinates instead of $r$ but such coordinates are more convenient.

We begin with a technical lemma. Let $U_{r+1}$ be the standard representation of $\mathfrak{g}$ in the $(r+1)$-dimensional space.

Lemma. $r\left(U_{r+1}\right)=2$.

Proof. Obviously, the weights of $U_{r+1}$ are $\beta_{j}=(0, \ldots, 0,1,0, \ldots, 0)$ with a 1 in the $j$ th slot. Set $x^{\beta_{j}}=x_{j}$. Using Table 1 , we obtain:

$$
R_{U_{r+1}}(x)=\sum x_{i_{0}}^{0} \ldots x_{i_{r}}^{r}\left(x_{0}+x_{1}+\ldots+x_{r}\right) .
$$


Simplifying, we get

$$
R_{U_{r+1}}(x)=\sum x_{i_{0}}^{0} \ldots x_{i_{r-1}}^{r-1} x_{i_{r}}^{r+1}+2 \sum_{0 \leqslant k \leqslant r-1 ; i_{k}<i_{k+1}} x_{i_{0}}^{0} \ldots x_{i_{k-1}}^{k-1} x_{i_{k}}^{k+1} x_{i_{k+1}}^{k+1} x_{i_{k+2}}^{k+2} \ldots \cdot x_{i_{r}}^{r} .
$$

So $\max r_{\nu}\left(U_{r+1}\right)=2$.

\section{3}

According to Propositions 5.5 and 5.4, we have:

Corollary. Any $\mathfrak{b}$-subquotient $U_{r+1}$ is a blue module. Moreover, if $V$ is a subquotient of $U_{r+1}$ and $r_{\nu}(V)=2$, then $H_{\nu}^{*}(V)=0$.

\section{4}

Now, let us return to the module $\mathfrak{n}$. We will have to consider also the modules $\mathfrak{g}$ and $\mathfrak{b}$, and the modules $\mathfrak{b}_{-}=\mathfrak{g} / \mathfrak{n}, \mathfrak{n}_{-}=\mathfrak{g} / \mathfrak{b}$ and $\mathfrak{h}=\mathfrak{b} / \mathfrak{n}$. Obviously, $\mathfrak{g}$ is an irreducible $\mathfrak{g}$-module and all the other modules are its subquotients. We have also the isomorphisms of $\mathfrak{h}$-modules:

$$
\mathfrak{g} \cong \mathfrak{n}_{-} \oplus \mathfrak{h} \oplus \mathfrak{n}, \quad \mathfrak{b}=\mathfrak{h} \oplus \mathfrak{n}, \quad \mathfrak{b}_{-} \cong \mathfrak{n}_{-} \oplus \mathfrak{h} .
$$

We see from formula (1.4) that it suffices to find the generating functions for $\mathfrak{n}_{-}, \mathfrak{h}$, and $\mathfrak{n}$.

The only weight of $\mathfrak{h}$ is zero, so $x^{\nu}=1$.

Now, if $\nu$ is a weight of $\mathfrak{n}$ or $\mathfrak{n}_{-}$, then $x^{\nu}=x_{i} x_{j}^{-1}$ for $i \neq j$, and $\nu$ is the weight of $\mathfrak{n}$ if and only if $i>j$, see [7]. Hence,

$$
\begin{gathered}
A_{\mathfrak{n}}(x)=\sum_{i>j} x_{i} x_{j}^{-1} ; \\
A_{\mathfrak{h}}(x)=r ; \\
A_{\mathfrak{n}_{-}}(x)=\sum_{i>j} x_{i}^{-1} x_{j}=A_{\mathfrak{n}}\left(x^{-1}\right) .
\end{gathered}
$$

Now, consider the functions $R_{V}(x)$ for $V \in\left\{\mathfrak{g}, \mathfrak{n}, \mathfrak{h}, \mathfrak{n}_{-}\right\}$. Recall that $L_{\nu}=\{j \mid \nu=$ $\left.w \rho+\beta_{j}\right\}$ for any $\nu$ (Proposition 4.13), or, equivalently, $x^{w \rho} x^{\beta_{j}}=x^{\nu}$. We know (Table 1) that $x^{w \rho}=x_{i_{0}}^{0} \ldots x_{i_{r}}^{r}$ and either $x^{\beta_{j}}=x_{k} x_{1}^{-1}$ or $x^{\beta_{j}}=1$. Multiplying these, we obtain the following monomials:

1) $x_{i_{0}}^{0} x_{i_{1}}^{1} \ldots x_{i_{r}}^{r}$.

2) $x_{i_{0}}^{-1} x_{i_{1}}^{1} \ldots x_{i_{r-1}}^{r-1} x_{i_{r}}^{r+1}$.

3) $x_{i_{0}}^{0} \ldots x_{i_{k-1}}^{k-1} x_{i_{k}}^{k} x_{i_{k+1}}^{k} x_{i_{k+2}}^{k+2} \ldots x_{i_{r-1}}^{r-1} x_{i_{r}}^{r+1}$ for $0 \leqslant k \leqslant r-2$.

In what follows, for brevity, we will not write explicitly the "normal" factors, i.e., factors $x_{i_{j}}^{k}$ with $k=j$.

4) $x_{i_{0}}^{-1} \ldots x_{i_{k}}^{k+1} x_{i_{k+1}}^{k+1} \ldots$ for $1 \leqslant k \leqslant r-1$.

5) $\ldots x_{i_{k-1}}^{k} x_{i_{k}}^{k} x_{i_{k+1}}^{k} \ldots$ for $1 \leqslant k \leqslant r-1$.

6) $\ldots x_{i_{k-1}}^{k} x_{i_{k}}^{k} \ldots x_{i_{l}}^{l} x_{i_{l+1}}^{l} \ldots$ for $1 \leqslant k \leqslant r$ and $0 \leqslant l \leqslant r-1$ such that $\{k-$ $1, k\} \cap\{l, l+1\}=\emptyset$. 
It is important to note that in cases 3,4 we can assume that $i_{k}<i_{k+1}$; similarly, in case 5 we will assume $i_{k-1}<i_{k}<i_{k+1}$ and in case $\mathbf{6}$ that $i_{k-1}<i_{k}$ and $l_{l}<i_{l+1}$. We do not assume that $k<l$.

\section{5}

Let us study the $\nu$-multiplicity of the above mentioned modules for any $\nu$ of types $\mathbf{1}-\mathbf{6}$.

1) One can obtain the monomial $x_{i_{0}}^{0} \ldots x_{i_{r}}^{r}$ by multiplying the same monomial by 1 for $V=\mathfrak{h}$ or by multiplying $\ldots x_{i_{k+1}}^{k} x_{i_{k}}^{k+1} \ldots$ by $x_{i_{k+1}} x_{i_{k}}^{-1}$ (here $0 \leqslant k \leqslant r-1$ and $V$ is isomorphic to $\mathfrak{n}$ if $i_{k+1}>i_{k}$ or $\mathfrak{n}_{-}$otherwise). It follows that $r_{\nu}(\mathfrak{h})=r$ and $r_{\nu}\left(\mathfrak{n}_{-}\right)+r_{\nu}(\mathfrak{n})=r$. Since $\operatorname{dim} H_{\nu}^{*}(\mathfrak{n})=1$ and $\mathfrak{h} \simeq \mathbb{C} \oplus \cdots \oplus \mathbb{C}$, as $\mathfrak{b}$-module, it follows that $\operatorname{dim} H_{\nu}^{*}(\mathfrak{n} ; \mathfrak{h})=r \operatorname{dim} H_{\nu}^{*}(\mathfrak{n})=r$. Now Proposition 5.7 shows that both $\mathfrak{n}$ and $\mathfrak{n}_{-}$are $\nu$-blue.

Clearly, if $\nu$ is of types $\mathbf{2}-\mathbf{6}$, then $r_{\nu}(\mathfrak{h})=0$.

Now, let $X_{\nu}=\left\{x^{\beta} \mid \in \beta \in L_{\nu}\right\}$. Hence, we have a one-to-one correspondence between $L_{\nu}$ and $X_{\nu}$ (this does not hold for $\mathfrak{h}$, because the multiplicity of the weight zero is greater than 1), so $r_{\nu}(V)=\operatorname{card} X_{\nu}$, where $\nu$ is of types 2-6 and $V \in$ $\left\{\mathfrak{n}, \mathfrak{n}_{-}, \mathfrak{g}\right\}$.

2) $x_{i_{0}}^{-1} \ldots x_{i_{r}}^{r+1}=x_{i_{0}}^{0} \ldots x_{i_{r}}^{r}\left(x_{i_{0}}^{-1} x_{i_{r}}\right)$. Obviously, this is the only way to get this monomial; so $X_{\nu}=\left\{x_{i_{0}}^{-1} x_{i_{r}}\right\}$, and $r_{\nu}(\mathfrak{g})=1$.

3) Clearly, $\ldots x_{i_{k}}^{k} x_{i_{k+1}}^{k} \ldots x_{i_{r}}^{r+1}$ can be obtained by multiplying $x^{w \rho}$ by $x_{i_{k}}^{-1} x_{i_{r}}$ or by $x_{i_{k+1}}^{-1} x_{i_{r}}$. So $r_{\nu}(\mathfrak{g})=$ card $\left\{x_{i_{k}}^{-1} x_{i_{r}}, x_{i_{k+1}}^{-1} x_{i_{r}}\right\}=2$.

4) is similar to 3$)$. So $X_{\nu}=\left\{x_{i_{0}}^{-1} x_{i_{k}}, x_{i_{0}}^{-1} x_{i_{k+1}}\right\}$, and $r_{\nu}(\mathfrak{g})=2$.

5) Here $X_{\nu}=\left\{x_{j_{1}}^{-1} x_{j_{1}}\right\}$, where $j_{1}, j_{2} \in\left\{i_{k-1}, i_{k}, i_{k+1}\right\}$. So $r_{\nu}(\mathfrak{g})=\operatorname{card} X_{\nu}=6$.

6) $X_{\nu}=\left\{x_{j_{1}}^{-1} x_{j_{1}}\right\}$, where $j_{2} \in\left\{i_{k-1}, i_{k}\right\}$ and $j_{1} \in\left\{i_{l}, i_{l+1}\right\}$. So $r_{\nu}(\mathfrak{g})=4$.

6.6. Lemma. $\mathfrak{n}$ is $\nu$-blue if $\nu$ is of types $\mathbf{1}-\mathbf{4}$.

Proof. Lemma was already proved for $\nu$ of type $\mathbf{1}$. For the types $\mathbf{2}-\mathbf{4}$ it is a corollary of Proposition 5.5.

\section{7}

We have not yet proved the theorem for the types $\mathbf{5}$ and $\mathbf{6}$. Here we will deal with type 5 .

Let $x^{\nu}=\ldots x_{i_{k-1}}^{k} x_{i_{k}}^{k} x_{i_{k+1}}^{k} \ldots$, where $i_{k}<i_{k}<i_{k+1}$. If $x^{\nu}=x^{w \rho} x^{\beta}$, then there are three weights $\beta$ which belong to $\mathfrak{n}$ :

1) $x^{\beta}=x_{i_{k-1}}^{-1} x_{i_{k}}$;

2) $x^{\beta}=x_{i_{k}}^{-1} x_{i_{k+1}}$;

3) $x^{\beta}=x_{i_{k-1}}^{-1} x_{i_{k+1}}$.

Thus, $x^{w \rho}$ is, respectively,

$$
\begin{gathered}
\ldots x_{i_{k}}^{k-1} x_{i_{k+1}}^{k} x_{i_{k-1}}^{k+1} \ldots \\
\ldots x_{i_{k+1}}^{k-1} x_{i_{k-1}}^{k} x_{i_{k}}^{k+1} \ldots \\
\ldots x_{i_{k+1}}^{k-1} x_{i_{k}}^{k} x_{i_{k-1}}^{k+1} \ldots
\end{gathered}
$$


Let $s$ be the number of inversions in the transposition $\left(i_{0} \ldots i_{r}\right)$. Then $\left(i_{0} \ldots i_{k} i_{k+1} i_{k-1} \ldots i_{r}\right)$ has $s+2$ inversions. So if $x^{w \rho}$ is given by $(6.5)$, then $l(w)=$ $s+2$. Similarly, $l(w)=s+2$ for $(6.6)$ and $l(w)=s+3$ for (6.7). This means that $\operatorname{dim} E_{1 \nu}^{s+2, *}(\mathfrak{n} ; \mathfrak{n})=2$ and $\operatorname{dim} E_{1 \nu}^{s+3, *}(\mathfrak{n} ; \mathfrak{n})=1$. So there are two possibilities:

either $\operatorname{dim} H_{\nu}^{s+2}(\mathfrak{n} ; \mathfrak{n})=2$ and $\operatorname{dim} H_{\nu}^{s+3}(\mathfrak{n} ; \mathfrak{n})=1$;

or $\quad \operatorname{dim} H_{\nu}^{s+2}(\mathfrak{n} ; \mathfrak{n})=1$ and $\operatorname{dim} H_{\nu}^{s+3}(\mathfrak{n} ; \mathfrak{n})=0$.

But similar considerations show that $\operatorname{dim} E_{1 \nu}^{1, *}\left(\mathfrak{n} ; \mathfrak{n}_{-}\right)= \begin{cases}2 & \text { if } l=s+1, \\ 1 & \text { if } l=s, \\ 0 & \text { if } l \neq s, s+1 .\end{cases}$

In particular, $E_{1 \nu}^{s+2, *}\left(\mathfrak{n} ; \mathfrak{n}_{-}\right)=0$ and so $H_{\nu}^{s+2}\left(\mathfrak{n} ; \mathfrak{n}_{-}\right)=0$. Since $E_{1 \nu}^{*}(\mathfrak{n} ; \mathfrak{h})=0$, we have $H_{\nu}^{s+2}\left(\mathfrak{n} ; \mathfrak{b}_{-}\right)=H_{\nu}^{s+2}\left(\mathfrak{n} ; \mathfrak{n}_{-}\right)=0$.

Now it remains to use the exact sequence

$$
0 \longrightarrow \mathfrak{n} \longrightarrow \mathfrak{g} \longrightarrow \mathfrak{b}_{-} \longrightarrow 0
$$

We see that $H_{\nu}^{*}(\mathfrak{n} ; \mathfrak{g})=0$ because $r_{\nu}(\mathfrak{g})=6 \neq 1$, and the corresponding cohomology sequence

$$
\ldots \longrightarrow H^{s+2}\left(\mathfrak{n} ; \mathfrak{b}_{-}\right) \longrightarrow H_{\nu}^{s+3}(\mathfrak{n} ; \mathfrak{n}) \longrightarrow H_{\nu}^{s+3}(\mathfrak{n} ; \mathfrak{g}) \longrightarrow \ldots
$$

shows that $H_{\nu}^{s+3}(\mathfrak{n} ; \mathfrak{n})=0$. Thus, the case 2$)$ takes place and

$$
\operatorname{dim} H_{\nu}^{*}(\mathfrak{n} ; \mathfrak{n})=\operatorname{dim} H_{\nu}^{s+2}(\mathfrak{n} ; \mathfrak{n})=1 .
$$

Hence, $\mathfrak{n}$ is $\nu$-blue by Proposition $4.3(\mathrm{v})$.

\section{8}

Finally, let $\nu$ be of type $\mathbf{6}, x=\ldots x_{i_{k-1}}^{k} x_{i_{k}}^{k} \ldots x_{i_{l}}^{l} x_{i_{l+1}}^{l} \ldots$.. We have to study 6 subcases:
(a) $i_{k-1}<i_{k}<i_{l}<i_{l+1}$
(b) $\quad i_{k-1}<i_{l}<i_{k}<i_{l+1}$
(d) $\quad i_{l}<i_{k-1}<i_{k}<i_{l+1}$
(c) $\quad i_{k-1}<i_{l}<i_{l+1}<i_{k}$
(e) $\quad i_{l}<i_{k-1}<i_{l+1}<i_{k}$
(f) $\quad i_{l}<i_{l+1}<i_{k-1}<i_{k}$

Recall that we have assumed that $i_{l}<i_{l+1}, i_{k-1}<i_{k}$. The elements of

$$
X_{\nu}=\left\{x_{i_{k-1}} x_{i_{l}}^{-1}, x_{i_{k}} x_{i_{l}}^{-1}, x_{i_{k-1}} x_{i_{l+1}}^{-1}, x_{i_{k}} x_{i_{l+1}}^{-1}\right\}
$$

belong partly to $X_{\nu}(\mathfrak{n})$ and partly to $X_{\nu}\left(\mathfrak{n}_{-}\right)$, namely:

(a) all the weights belong to $\mathfrak{n}_{-}$, so $r_{\nu}(\mathfrak{n})=0$, and $r_{\nu}\left(\mathfrak{n}_{-}\right)=4$.

(b) $\nu$ belongs to $\mathfrak{n}$ if and only if $x=x_{i_{k}} x_{i_{l}}^{-1}$; the remaining three weights belong to $\mathfrak{n}_{-}$and so $r_{\nu}(\mathfrak{n})=1$, while $r_{\nu}\left(\mathfrak{n}_{-}\right)=3$.

(c) $X_{\nu}\left(\mathfrak{n}_{-}\right)=\left\{x_{i_{k}} x_{i_{l}}^{-1}\right.$, and $\left.x_{i_{k}} x_{i_{l+1}}^{-1}\right\}$; hence, $r_{\nu}(\mathfrak{n})=2$, and $r_{\nu}\left(\mathfrak{n}_{-}\right)=2$.

(d) $X_{\nu}\left(\mathfrak{n}_{-}\right)=\left\{x_{i_{k}} x_{i_{l}}^{-1}\right.$, and $\left.x_{i_{k-1}} x_{i_{l+1}}^{-1}\right\}$, hence, $r_{\nu}(\mathfrak{n})=r_{\nu}\left(\mathfrak{n}_{-}\right)=2$.

The cases (e) and (f) are opposite to (b) and (a), respectively, so we have:

$$
\text { (e) } \quad r_{\nu}(\mathfrak{n})=3 \text { and } r_{\nu}\left(\mathfrak{n}_{-}\right)=1 \quad(f) \quad r_{\nu}(\mathfrak{n})=4 \text { and } r_{\nu}\left(\mathfrak{n}_{-}\right)=0 .
$$

Clearly, $r_{\nu}\left(\mathfrak{n}_{-}\right)=c r_{\nu}(\mathfrak{n} ; \mathfrak{g})$. So, by Proposition $5.4, \mathfrak{n}$ is $\nu$-blue in cases (a), (b), (e) and (f). 


\section{9}

It remains to study cases $6(\mathrm{c}), \mathbf{6}(\mathrm{d})$. Let $\nu$ be of type $\mathbf{6}(\mathrm{c})$. The corresponding to $X_{\nu}(\mathfrak{n})$ eigenvectors in $\mathfrak{n}$ are the elementary matrices $E_{i_{k} i_{l}}$ and $E_{i_{k} i_{l+1}}$.

Now let $V_{j}$ be the $\mathfrak{b}$-submodule of $\mathfrak{n}$ which consists of matrices with first $j$ rows zero and $\tilde{V}_{j}=V_{j-1} / V_{j}$. We may identify this subquotient with the linear space of matrices with all the rows zero, except the $j$ th row. So this $\mathfrak{n}$-module is isomorphic to $U_{r+1}^{\prime}$, cf. sec. 6.2. To demonstrate the $\mathfrak{b}$-isomorphism of these modules, one has to add the constant weight $\lambda$ such that $x^{\lambda}=x_{j}$.

We see that $r_{\nu}(\mathfrak{n})=r_{\nu}\left(\tilde{V}_{i_{k}}\right)=2$. Obviously, Lemma 6.2 holds for the dual module $U_{r+1}^{\prime}$, so $H_{\nu}^{*}\left(\mathfrak{n} ; \tilde{V}_{i_{k}}\right)=0$. Since $\operatorname{dim} E_{1 \nu}^{*}(\mathfrak{n} ; \mathfrak{n})=\operatorname{dim} E_{1 \nu}^{*}\left(\mathfrak{n} ; \tilde{V}_{i_{k}}\right)$, they are isomorphic. So $H_{\nu}^{*}(\mathfrak{n} ; \mathfrak{n})=H_{\nu}^{*}\left(\mathfrak{n} ; \tilde{V}_{i_{k}}\right)=0$, and $\mathfrak{n}$ is $\nu$-blue.

Similar arguments (take columns instead of rows) show that $\mathfrak{n}$ is $\nu$-blue if $\nu$ is of type $6(d)$.

Thus, we proved Theorem 6.1 for all the $\nu$.

6.10. Corollary. $\mathfrak{b}-\mathfrak{n}$-modules $\mathfrak{b}, \mathfrak{n}_{-}$, and $\mathfrak{b}_{-}$are blue.

Proof. As follows from (6.4), $\mathfrak{n}_{-}$is isomorphic to $\mathfrak{n}^{\prime}, \mathfrak{b}_{-}$is isomorphic to $\mathfrak{g} / \mathfrak{n}$, and $\mathfrak{n}_{-} \cong \mathfrak{g} / \mathfrak{b}$. Now apply Propositions 5.6, 5.8.

\section{§7. Dimensions of the cohomology with the coefficients in the adjoint module}

\section{1}

In $\delta \S 7$ and 8 we still assume that $\mathfrak{g}=A_{r}$ and preserve the notations of the previous sections. We will also need many new notations: for any $w=\left(\begin{array}{ccc}0 & \ldots & r \\ i_{0} & \ldots & i_{r}\end{array}\right)$ set

$$
\begin{aligned}
& h_{\mathbf{1}}(w)=\left|\left\{j \mid i_{j}<i_{j+1}\right\}\right| ; \\
& h_{\mathbf{2}}(w)= \begin{cases}1 & \text { if } i_{0}<i_{r}, \\
0 & \text { if } i_{0}>i_{r} ;\end{cases} \\
& h_{\mathbf{3}}(w)=\left|\left\{j \mid i_{j}<i_{r}<i_{j+1}\right\}\right| ; \\
& h_{\mathbf{4}}(w)=\left|\left\{j \mid i_{j}<i_{0}<i_{j+1}\right\}\right| ; \\
& h_{\mathbf{5}}(w)=\left|\left\{j \mid i_{j-1}<i_{j}<i_{j+1}\right\}\right| ; \\
& h_{\mathbf{6}}(w)=\left|\left\{(k, l) \mid i_{k-1}<i_{l}<i_{k}<i_{l+1}\right\}\right|=\left|\left\{(k, l) \mid i_{l}<i_{k-1}<i_{l+1}<i_{k}\right\}\right| .
\end{aligned}
$$

Further, for any $\mathbf{N} \in\{\mathbf{1}, \ldots, \mathbf{6}\}$ set $h_{\mathbf{N}}=\sum_{w \in W} h_{\mathbf{N}}(w)$.

Finally, let $W_{l}=\{w \in W \mid l(w)=l\}$, and denote: $h_{\mathbf{N}}(l)=\sum_{N \in W_{l}} h_{\mathbf{N}}(w)$. For $l<0$ set $h_{\mathbf{N}}(l)=0$.

7.2. Theorem. $\operatorname{dim} H^{*}(\mathfrak{n} ; \mathfrak{n})=h_{\mathbf{1}}+h_{\mathbf{2}}+h_{\mathbf{3}}+h_{\mathbf{4}}+h_{\mathbf{5}}+2 h_{\mathbf{6}}$.

\subsection{Theorem.}

$$
\begin{aligned}
& \operatorname{dim} H^{k}(\mathfrak{n} ; \mathfrak{n})=h_{\mathbf{1}}(k-1)+h_{\mathbf{2}}(k)+h_{\mathbf{3}}(k-1)+ \\
& h_{\mathbf{4}}(k-1)+h_{\mathbf{5}}(k-2)+h_{\mathbf{6}}(k-2)+h_{\mathbf{6}}(k-1) .
\end{aligned}
$$




\subsection{Proof of Theorems 7.2, 7.3}

Consider, for example, type $\mathbf{1}$. If

$$
x^{\nu}=x_{i_{0}}^{0} \ldots x_{i_{r}}^{r}=x_{j} \cdot x_{k}^{-1} \cdot x^{w \rho}, \text { where } x^{\beta j}=x_{j} \cdot x_{k}^{-1} \in \mathfrak{n},
$$

then, as we have seen, $j=i_{s+1}, k=i_{s}$ and $j>k$. So $w \in W_{l+1}$ for all $j, k$, and the number of admissible pairs $(j, k)$ is equal to the number of indices $s$ such that $i_{s+1}>i_{s}$, i.e., to $h_{\mathbf{1}}(w)$. So we see that $\sum_{\nu} \operatorname{dim} H_{\nu}^{*}$, where $\nu$ runs over type $\mathbf{1}$ vectors, is equal to $h_{\mathbf{1}}$. If we want to find $\sum_{\nu} \operatorname{dim} H_{\nu}^{k}$, we must take $l+1=k$, so $w \in W_{k-1}$.

Similar considerations for other types lead us to Table 2. Theorems 7.2, 7.3 are its direct consequences.

\section{5}

We have subdivided the set of weights into several subsets according to their types. Set $H_{\mathbf{N}}^{*}=\underset{\nu \in \mathbf{N}}{\oplus} H_{\nu}^{*}$ for any type $\mathbf{N}$ from Table 2. Let us find $\operatorname{dim} H_{\mathbf{N}}^{*}$.

1) for $w=\left(\begin{array}{ccc}0 & \ldots & r \\ i_{0} & \ldots & i_{r}\end{array}\right)$, set $\bar{w}=\left(\begin{array}{cccc}0 & 1 & \ldots & r \\ i_{r} & i_{r-1} & \ldots & i_{0}\end{array}\right)$. Then, clearly, $h_{\mathbf{1}}(\bar{w})=$ $r-h_{\mathbf{1}}(w)$. Since the map $w \mapsto \bar{w}$ is a one-to-one,

$$
h_{\mathbf{1}}=\sum_{w \in W} h_{\mathbf{1}}(w)=\sum_{w \in W} h_{\mathbf{1}}(\bar{w})=\sum_{w \in W}\left(r-h_{\mathbf{1}}(w)\right)=r \cdot|W|-h_{\mathbf{1}} ;
$$

hence,

$$
\operatorname{dim} H_{\mathbf{1}}^{*}(\mathfrak{n} ; \mathfrak{n})=h_{\mathbf{1}}=\frac{1}{2} r \cdot(r+1) !
$$

2) Clearly, $i_{0}<i_{r}$ in the half of the cases, hence,

$$
\operatorname{dim} H_{2}^{*}(\mathfrak{n} ; \mathfrak{n})=h_{2}=\frac{1}{2}|W|=\frac{(r+1) !}{2}
$$

3) Let us fix $j$. Then $\left|\left\{w \mid i_{j}<i_{r}<i_{j+1}\right\}\right|=\frac{1}{6}|W|$. So $\operatorname{dim} H_{\mathbf{3}}^{*}=h_{\mathbf{3}}$ is equal to the sum of such numbers for all $j$ such that $1 \leqslant j \leqslant r-1$. Hence,

$$
\operatorname{dim} H_{\mathbf{3}}^{*}(\mathfrak{n} ; \mathfrak{n})=h_{\mathbf{3}}=\sum_{1 \leqslant j \leqslant r-1} \frac{|W|}{6}=\frac{(r-1)}{6} \cdot(r+1) !
$$

4), 5) Obviously $h_{\mathbf{3}}=h_{\mathbf{4}}$ (moreover, $h_{\mathbf{3}}(k)=h_{\mathbf{4}}(k)$ for any $k$ ), hence,

$$
\operatorname{dim} H_{\mathbf{4}}^{*}(\mathfrak{n} ; \mathfrak{n})=h_{\mathbf{4}}=\frac{(r-1)}{6} \cdot(r+1) !
$$

The same formula is true for $H_{5}$ :

$$
\operatorname{dim} H_{\mathbf{5}}^{*}(\mathfrak{n} ; \mathfrak{n})=h_{\mathbf{5}}=\frac{(r-1)}{6} \cdot(r+1) !
$$

because in all the cases $\mathbf{3}-\mathbf{5}$ we have to order 3 indices in the transposition.

6) Similarly, fix $k$ and $l$. Then there are $\frac{1}{24}|W|$ transpositions which satisfy the 
condition $i_{k-1}<i_{l}<i_{k}<i_{l+1}$. So

$$
\operatorname{dim} H_{\mathbf{6}(b)}^{*}=\sum_{(k, l)} \frac{|W|}{24}=\operatorname{dim} H_{\mathbf{6}(e)}^{*} .
$$

There are $(r-1) \cdot(r-2)$ pairs $(k, l)$ such that $\{k-1, k\} \cap\{l, l-1\}=\emptyset$. So

$$
\operatorname{dim} H_{\mathbf{6}}^{*}(\mathfrak{n} ; \mathfrak{n})=2 h_{\mathbf{6}}=\frac{(r-1)(r-2)}{12}(r+1) !
$$

7.6. Theorem. $\operatorname{dim} H^{*}(\mathfrak{n} ; \mathfrak{n})=\frac{1}{12}\left(r^{2}+9 r+2\right)(r+1)$ !.

Proof: formulas (7.2)-(7.7).

7.7

From Theorem 7.3 one can deduce $\operatorname{dim} H^{k}(\mathfrak{n} ; \mathfrak{n})$ for small $k$ :

\section{Theorem.}

$$
\operatorname{dim} H^{k}(\mathfrak{n} ; \mathfrak{n})= \begin{cases}1 & \text { if } k=0 \text { and } r>0 \\ 2 r & \text { if } k=1 \text { and } r>1 \\ \left(3 r^{2}+3 r-4\right) / 2 & \text { if } k=2 \text { and } r>2 \\ \frac{2}{3} r^{3}+2 r^{2}-\frac{11}{3} r-1 & \text { if } k=3 \text { and } r>3\end{cases}
$$

If $r=2$, then $\operatorname{dim} H^{2}=5$, $\operatorname{dim} H^{3}=2$. If $r=3$, then $\operatorname{dim} H^{3}=21$.

Proof of this theorem also consists in studying different types of transpositions with 1, 2 or 3 inversions. It is trivial, but cumbersome, so we omit it.

$\S 8$. The behavior of $h(r, k)=\operatorname{dim} H^{k}(\mathfrak{n} ; \mathfrak{n})$ as a function of $k$ and $r=\mathrm{rkg}$

8.1. Theorem. If $k$ is fixed and $r$ is sufficiently great, then for some rational in $k$ functions $c_{l}(k)$ we have

$$
h(r, k)=\sum_{0 \leqslant l \leqslant k} c_{l}(k) \cdot r^{k-1} .
$$

Proof will be given in sec. 8.2-8.8. In sec. 8.2-8.6 we fix a permutation $w=$ $\left(\begin{array}{ccc}0 & \ldots & r \\ i_{0} & \ldots & i_{r}\end{array}\right)$. Let $l=l(w)$ and $X=\{0,1, \ldots, r\}$.

\section{2}

Let $\sigma_{i}=\left(\begin{array}{cccccc}0 & \ldots & i-1 & i & \ldots & r \\ 0 & \ldots & i & i-1 & \ldots & r\end{array}\right)$, where $i=1, \ldots, r$, be the standard generators of $W$. By definition of length, $w$ is a product of $l$ generators: $w=$ $\sigma_{i_{1}} \sigma_{i_{2}} \ldots \sigma_{i_{l}}$. Let $K=\left\{i_{1}, \ldots, i_{l}\right\}$. Let us subdivide $K$ into subsets $K_{1}, \ldots, K_{m}$ so that

(i) if $\left|i_{a}-i_{b}\right| \leqslant 1$ (in particular, if $i_{a}=i_{b}$ ), then $i_{a}, i_{b}$ belong to the same $K_{j}$; 
(ii) if on the contrary, there is an integer $i$ such that $i_{a}<i<i_{b}$ and none of $i_{j}$ is equal to $i$, then $i_{a}$ and $i_{b}$ belong to distinct sets $K_{j}$.

Clearly, (i) and (ii) define the subsets $K_{t}$ uniquely, up to permutation. It is well known that if $i$ and $j$ belong to distinct sets $K_{t}$, then $\sigma_{i} \sigma_{j}=\sigma_{j} \sigma_{i}$. Thus we can write

$$
w=w_{1} w_{2} \ldots w_{m}
$$

where $w_{j}$ is the product of the generators which belong to $K_{j}$. Such a representation of $w$ is a canonical one, and $w_{i} w_{j}=w_{j} w_{i}$ for all $i, j$.

\section{3}

Let us fix $j$ and consider $K_{j}$ and the corresponding permutation $w_{j}$. If follows from the definition of $K_{j}$ that there exist integers $k<l$ such that $K_{j}$ contains $\sigma_{i}$ (perhaps, more than once) if and only if $k<i \leqslant l$ and the segments $[k, l]$ have no common elements for distinct sets $K_{j}$. We will call $B_{j}=\{k, k+1, \ldots, l\}$ the block corresponding to $K_{j}$. One easily sees that $B_{j}$ is $w$-invariant and that

$$
w_{j}(i)=\left\{\begin{array}{ll}
w(i) & \text { if } i \in B_{j} \\
i & \text { if } i \notin B_{j}
\end{array} .\right.
$$

So $w$ and $w_{j}$ give the same permutation of $\{k, \ldots, l\}$. Let us denote by $w_{B}$ the corresponding permutation of $\{0, \ldots, l-k\}$; so

$$
w_{B}=\left(\begin{array}{ccc}
0 & \ldots & l-k \\
i_{k}-k & \ldots & i_{l}-k
\end{array}\right) \text {. }
$$

8.4. Lemma. Let $B_{1}, \ldots, B_{m}$ be the blocks of $w$, then

i) $l\left(w_{B_{j}}\right) \geqslant \frac{1}{2} \operatorname{card} B_{j}$ for any $j$.

ii) $l(w)=\sum_{1 \leqslant j \leqslant m} l\left(w_{B_{j}}\right)$.

Proof. Since $w=\sigma_{i_{1}} \ldots \sigma_{i_{l}}$ is a minimal representation of $w$ as the product of generators, we easily deduce that $i \in X$ belongs to some block if and only if $i$ forms an inversion with some $i_{1}$ from the same block. This gives us (i).

Now, the elements of distinct blocks do not form inversions, and the number of inversions in the restriction of $w$ onto $B$ and in $w_{B}$ coincide. This implies (ii).

8.5. Lemma. Let $B_{1}, \ldots, B_{m}$ be the blocks of $w$, and $r_{j}=\operatorname{card} B_{j}$. Then

$$
h_{\mathbf{1}}(w)=r+m-\sum_{1 \leqslant j \leqslant m} r_{j}+\sum_{1 \leqslant j \leqslant m} h_{\mathbf{1}}\left(w_{B_{j}}\right) .
$$

Proof. Let $Z=\left\{j \mid i_{j}<i_{j+1}\right\}$, so $h_{1}(w)=|Z|$. Let $Z_{1}=X \backslash \cup B_{j}$ and let $Z_{2}$ be the set of last elements of blocks: $i \in Z_{2}$ if and only if there exists a $B_{j}$ equal to $\{k, \ldots, i\}$. Then, clearly

$$
\left|Z_{1}\right|=r+1-\sum_{1 \leqslant j \leqslant m} r_{j}, \quad\left|Z_{2}\right|=m, \quad Z_{1} \cap\left(Z \cup Z_{2}\right)=\left(Z_{1} \cup Z_{2}\right) \backslash\{r\}
$$


and it is always true that $r \in Z_{1} \cup Z_{2}$. Finally, let $i \in Z, i \notin Z_{1} \cup Z_{2}$. Then $i$ and $i+1$ belong to some block $B=\{k, \ldots, l\}$. So

$$
\left|Z_{1} \backslash\left(Z \cup Z_{2}\right)\right|=\sum_{B} h_{1}\left(w_{B}\right) .
$$

Now (8.5) is a direct consequence of (8.6) and (8.7).

\section{6}

We can get similar formulas for functions $h_{\mathbf{2}}(w), \ldots, h_{\mathbf{6}}(w)$; for example, we see that if $r>2 l$, then $h_{\mathbf{2}}(w)=1$. We won't need the exact formulas, so we shall formulate the corresponding result as follows.

Call $B$ a block of type 1 if $B \ni 0$, of type 2 if $B \ni r$ or of type 3 if $0, r \notin B$, respectively. (Lemma 8.4 (i) implies that if $r>2 l$, then $B$ cannot simultaneously contain 0 and $r$.) Let $\Omega$ be the set of all the $w_{B}$ for given types of blocks. We will say that $\Omega$ is a structure on the Weyl group $W$, and that $w$ is a permutation of the structure $\Omega$.

Lemma. In what follows the $c_{i}$ depend only on $\Omega$ and do not depend on $w$ or $r$ :

$$
h_{\mathbf{2}}(w)=c_{2}=1, \quad h_{\mathbf{3}}(w)=c_{3}, \quad h_{\mathbf{4}}(w)=c_{4}, \quad h_{\mathbf{5}}(w)=r+c_{5}, \quad h_{\mathbf{6}}(w)=c_{6} .
$$

Proof is similar to that of Lemma 8.5, and we omit it.

Note, however, the difference between $h_{\mathbf{1}}, h_{\mathbf{5}}$ and the other four functions. This difference is due to the fact that if $i \notin \cup B_{j}$, then the inequalities $i_{i}<i_{i+1}$ and $i_{i-1}<i_{i}<i_{i+1}$ hold, and other inequalities in formulas (7.1) do not hold.

\section{7}

Let $\Omega$ be a structure on $W$, and $W(r, \Omega)$ the set of all permutations of $\{0, \ldots, r\}$ with the given structure $\Omega$. We will consider $f(r, \Omega)=|W(r, \Omega)|$ as a function of $r, \Omega$ for a fixed $\Omega$.

Lemma. $f(r, \Omega)=\sum_{1 \leqslant k \leqslant N} \alpha_{k} r^{k}$, where $\alpha_{N} \neq 0$ and $N$ is equal to the number of blocks of type 3 in $\Omega$.

Proof. First, suppose, that $\Omega=\left\{w_{1}, \ldots, w_{m}\right\}$, all the $w_{i}$ are of type 3 , and no two pairs of them coincide. Then there is a one-to-one correspondence between the permutations of the structure $\Omega$ and the arrangements of the sets $B_{1}, \ldots, B_{m}$ of given lengths $a_{1}, \ldots, a_{m}$ from $\{1, \ldots, r-1\}$. It is well-known that the number of such arrangements is equal to

$$
\frac{\left(r-1+m-\sum_{j} a_{j}\right) !}{\left(r-1-\sum_{j} a_{j}\right) !} .
$$

So it is a polynomial in $r$ of degree $m$. 
If we have blocks of type 1 or 2 , then their places are fixed, and we have to place the remaining blocks. So $f(r, \Omega)$ is still a polynomial in $r$ whose degree is equal to the number of blocks of type 3 .

Finally, if $w_{1}=w_{2}=\ldots=w_{b_{1}}, w_{b_{1}+1}=\ldots=w_{b_{1}+b_{2}}$, and so on, we have to divide (8.9) by $\prod_{i}\left(b_{i}\right)$ !.

\section{8}

Now we are ready to finish the proof of the theorem. Let $\Psi_{l}$ be the set of all structures with $l$ inversions. Lemma 8.4 shows that $\Psi_{l}$ is finite and does not depend on $r$ if $r$ is sufficiently large. We know (Lemmas 8.5, 8.6) that the functions $h_{i}(w)$ depend only on $\Omega$, i.e., $h_{i}(w)=h_{i}(\Omega)$.

So we may rewrite Theorem 7.3 so that every summand in it takes the form

$$
h_{i}(l)=\sum_{\Omega \in \Psi_{l}} h_{i}(\Omega) f(t, \Omega) .
$$

Every summand is a polynomial and their number does not depend on $r$. Applying Theorem 7.3 we complete the proof.

8.9. Theorem. Let $c_{l}(k)$ be the coefficients in (8.1). Then $(k-l) ! \cdot c_{l}(k)$ is a polynomial in $k$.

Proof. It is clear from formulas (8.10), (8.9), that $k ! \cdot c_{l}(k)$ is a polynomial. But if $l>k$ then the term of degree $k-l$ vanishes, so $c_{l}(k)=0$.

\subsection{0}

Let us find $c_{0}(k)$. Clearly, we must consider only two terms in Theorem 7.3:

$$
\operatorname{dim} H^{k}(\mathfrak{n} ; \mathfrak{n})=\sum_{\Omega \in \Psi_{l}} f(r, \Omega) \cdot h_{\mathbf{2}}(\Omega)+\sum_{\Omega \in \Psi_{l}} f(r, \Omega) \cdot h_{\mathbf{1}}(\Omega)+\ldots
$$

because the degrees of the terms denoted by dots are $<k$. We know, that $h_{\mathbf{2}}(\Omega)=1$ and $h_{\mathbf{1}}(\Omega)=r+C$. Hence, the terms in (8.11) have degree $k$ if and only if $\Omega$ consists of the maximal number of blocks ( $k$ for the first term and $k-1$ for the second one, respectively).

But then every block has only one inversion, so $\Omega=\left(\left(\begin{array}{ll}0 & 1 \\ 1 & 0\end{array}\right), \ldots,\left(\begin{array}{ll}0 & 1 \\ 1 & 0\end{array}\right)\right)$ and $|\Omega|$ is equal to $k$ or $k-1$. Then

$$
f(r, \Omega)=\left(\begin{array}{c}
r-|\Omega|+1 \\
|\Omega|
\end{array}\right)=\frac{r^{|\Omega|}}{|\Omega| !}+\ldots
$$

So

$$
c_{0}(k)=\frac{1}{k !}+\frac{1}{(k-1) !}=\frac{1}{k !} \cdot(k+1) .
$$

\subsection{1}

Using the same method, one can prove that

$$
c_{1}(k)=\frac{1}{(k-1) !} \cdot \frac{k^{2}-1}{2} ; \quad c_{2}(k)=\frac{1}{(k-2) !} \cdot \frac{3 k^{3}-k^{2}-92 k+116}{24} .
$$


For example,

$$
\operatorname{dim} H^{3}(\mathfrak{n} ; \mathfrak{n})=\frac{4}{3 !} r^{3}+\frac{4}{2 !} r^{2}-\frac{88}{24} r+\ldots
$$

which coincides with Theorem 7.7;

$$
\operatorname{dim} H^{4}(\mathfrak{n} ; \mathfrak{n})=\frac{5}{24} r^{4}+\frac{5}{4} r^{3}-\frac{19}{6} r^{2}+\ldots
$$

\section{$\S 9$. Some other results and problems}

\section{1}

The most natural generalization of the problems solved in this paper is to study $\mathfrak{g} \neq A_{r}$. I also studied the other classical algebras and $G_{2}$. Their study is much more complicated then that of $A_{r}$ because one has to consider more cases. The values of $\left|G_{\mathfrak{n}}\right|$ are given in Table 3.

Moreover, I know, that $\operatorname{dim} H^{*}(\mathfrak{n} ; \mathfrak{n})=\left|G_{\mathfrak{n}}\right|$ in all these cases except $\mathfrak{g}=D_{r}$. The main difficulty is that a straightforward analogue of Lemma 6.2 fails for $D_{r}$ (see below).

Problem. Calculate $\operatorname{dim} H^{*}(\mathfrak{n} ; \mathfrak{n})$ for $\mathfrak{g}=D_{r}$ as well as for the exceptional algebras.

\section{2}

Now let us discuss other modules. The classical Lie algebras $A_{r}, B_{r}, C_{r}, D_{r}$ have standard (identity) representations, whose dimensions are $r+1,2 r+1,2 r$ and $2 r$, respectively. Their multiplicities (see 3.9) are equal to 2 for the types $A, B, C$ and 4 for the type $D$. We will analyze these representations elsewhere; here I only give the main result. For simplicity we assume $\mathfrak{g}=A_{r}$, the same results hold for the types $B$ and $C$.

Let $v_{0}, \ldots, v_{r}$ be the standard basis of $U_{r+1}$. Then all the $\mathfrak{b}$-submodules are generated by $V_{i}=\operatorname{Span}\left(v_{i}, v_{i+1}, \ldots, v_{r}\right)$ for $0 \leqslant i \leqslant r$. Let $V_{i j}=V_{i} / V_{j+1}$; clearly, $\operatorname{dim} V_{i j}=j+1-i$. Set $l=\operatorname{dim} V_{i j}$. Then

$$
\operatorname{dim} H^{*}\left(\mathfrak{n} ; V_{i j}\right)=|W| \cdot \frac{l(r+2-l)}{r+1}
$$

and $\operatorname{dim} H^{k}\left(\mathfrak{n} ; V_{i j}\right)$ for a fixed $k$ does not depend on $i, j, l$ if $i>k, l>k, r-j>k$. This dimension is a polynomial in $r$ and its degree is equal to $k$.

It is worth to note that $\operatorname{dim} H^{*}$ grows as $|W| \cdot \operatorname{dim} V$ as $r \longrightarrow \infty$ and the growth of $\operatorname{dim} H^{k}$ is a polynomial one. Moreover, $\operatorname{dim} H^{*}$ has a single formula for all $r$, great and small, whereas $\operatorname{dim} H^{k}$ has such formula only for sufficiently large $r$.

\section{3}

Finally, Table 4 gives $\operatorname{dim} H\left(\mathfrak{n} ; \Lambda^{*} \mathfrak{n}\right)$ for different types of $\mathfrak{g}$.

Problem. Study the growth of $\operatorname{dim} H\left(\mathfrak{n} ; \Lambda^{*} \mathfrak{n}\right)$ as $r \longrightarrow \infty$ for various series of matrix algebras $\mathfrak{g}$; first, for $\mathfrak{g}=A_{r}$. 


\section{Table 1}

\begin{tabular}{|c|c|c|}
\hline $\mathfrak{g}$ & $\rho$ & $P(t, x)$ \\
\hline$A_{r}$ & $(12 \ldots r)$ & $\sum x_{i_{0}}^{0} x_{i_{1}}^{1} \ldots x_{i_{r}}^{r} t^{l(w)}$ \\
\hline$B_{r}$ & $\left(\frac{1}{2} \frac{3}{2} \frac{5}{2} \ldots \frac{2 r-1}{2}\right)$ & $\sum x_{i_{1}}^{ \pm 1 / 2} \ldots x_{i_{r}}^{ \pm(2 r-1) / 2} t^{l(w)}$ \\
\hline$C_{r}$ & $\left(12 \frac{2}{1} \ldots r\right)$ & $\sum x_{i_{1}}^{ \pm 1} \ldots x_{i_{r}}^{ \pm r} t^{l(w)}$ \\
\hline$D_{r}$ & $(012 \ldots \ldots(r-1))$ & $\sum x_{i_{1}}^{0} x_{i_{2}}^{ \pm 1} \ldots x_{i_{r}}^{ \pm(r-1)} t^{l(w)}$ \\
\hline
\end{tabular}

\section{Table 2}

\begin{tabular}{|c|c|c|c|c|c|c|}
\hline \multirow{2}{*}{\multicolumn{2}{|c|}{$\nu$}} & \multicolumn{4}{|c|}{$\operatorname{Dim} E_{1 \nu}^{k *}$ for different $\mathrm{k}$} & \multirow[t]{2}{*}{$H^{*}$} \\
\hline & & $k=l$ & $k=l+1$ & $k=l+2$ & $k=l+3$ & \\
\hline \multicolumn{2}{|l|}{ I } & 0 & $h_{I}(w)$ & 0 & 0 & $\operatorname{dim} H^{l+1}=h_{I}(w)$ \\
\hline & (a) $i_{0}<i_{r}$ & 1 & 0 & 0 & 0 & $\operatorname{dim} H^{l}=1$ \\
\hline & (b) $i_{0}>i_{r}$ & 0 & 0 & 0 & 0 & $H^{*}=0$ \\
\hline & (a) $i_{j}<i_{j+1}<i_{r}$ & 1 & 1 & 0 & 0 & $H^{*}=0$ \\
\hline & (b) $i_{j}<i_{r}<i_{j+1}$ & 0 & 1 & 0 & 0 & $\operatorname{dim} H^{l+1}=1$ \\
\hline & (c) $i_{r}<i_{j}<i_{j+1}$ & 0 & 0 & 0 & 0 & $H^{*}=0$ \\
\hline \multirow[t]{3}{*}{ IV } & (a) $i_{0}<i_{j}<i_{j+1}$ & 1 & 1 & 0 & 0 & $H^{*}=0$ \\
\hline & (b) $i_{0}<i_{j}<i_{j+1}$ & 0 & 1 & 0 & 0 & $\operatorname{dim} H^{l+1}=1$ \\
\hline & (c) $i_{0}<i_{j}<i_{j+1}$ & 0 & 0 & 0 & 0 & $H^{*}=0$ \\
\hline \multicolumn{2}{|l|}{$\mathrm{V}$} & 0 & 0 & 2 & 1 & $\operatorname{dim} H^{l+2}=1$ \\
\hline & (a) & 0 & 0 & 0 & 0 & $H^{*}=0$ \\
\hline & (b) & 0 & 0 & 1 & 0 & $\operatorname{dim} H^{l+2}=1$ \\
\hline & (c) & 0 & 1 & 1 & 0 & $H^{*}=0$ \\
\hline & (d) & 0 & 1 & 1 & 0 & $H^{*}=0$ \\
\hline & (e) & 0 & 2 & 1 & 0 & $\operatorname{dim} H^{l+1}=1$ \\
\hline & (f) & 1 & 2 & 1 & 0 & $H^{*}=0$ \\
\hline
\end{tabular}




\section{Table 3}

\begin{tabular}{|c|c|c|c|c|c|}
\hline $\mathfrak{g}$ & $A_{r}$ & $B_{r}$ & $C_{r}$ & $D_{r}$ & $G_{2}$ \\
\hline$\frac{1}{|W|}\left|G_{\mathfrak{n}}\right|$ & $\frac{r^{2}+9 r+2}{12}$ & $\frac{2 r^{2}+10 r-1}{12}$ & $\frac{2 r^{2}+8 r+3}{12}$ & $\frac{2 r^{2}+7 r+3}{12}$ & 34 \\
\hline
\end{tabular}

\section{Table 4}

\begin{tabular}{|c|c|c|c|c|c|}
\hline $\mathfrak{g}$ & $A_{1}$ & $A_{2}$ & $A_{3}$ & $B_{2}$ & $G_{2}$ \\
\hline $\operatorname{dim} H^{*}\left(\mathfrak{n} ; \Lambda^{*} \mathfrak{n}\right)$ & 4 & 36 & 600 & 68 & 220 \\
\hline
\end{tabular}

\section{References}

[1] Aribaud F., Une nouvelle démonstration d'un théorème de R. Bott et B. Kostant. (French) Bull. Soc. Math. France. 95, 1967, 205-242.

[2] Barut A. O., Raczka R., Theory of group representations and applications, Warszawa, 1977.

[3] Bernstein J. N., Gelfand I. M., Gelfand S. I., Structure of representations that are generated by vectors of highest weight. Funckcional. Anal. i Priložen. 5 (1971), no. 1, 1-9. (Russian; English translation in Functional Anal. Appl. 5 (1971), 1-8)

[4] Bernstein J. N., Gelfand I. M., Gelfand S. I., Differential operators on the base affine space and a study of $\mathfrak{g}$-modules. In: Gelfand I. M. (ed.) Summer school of the Janos Bolyai Math.Soc., Halsted Press, NY, 1975, 21-64

[5] Bott R., Homogeneous vector bundles, Ann. Math. 66, 1957, 203-248

[6] Bourbaki N., Groupes et algébres de Lie. Chapitres IV-VI, Paris, 1968.

[7] Bourbaki N., Groupes et algébres de Lie. Chapitres VII, VIII, Paris, 1975.

[8] Cartan H., Eilenberg S., Homological algebra, Princeton Univ. Press, 1956.

[9] Hochschild G., Serre J.P., Cohomology of Lie algebras, Ann. Math. 57, 1953, 591-603

[10] Jacobson N., Lie algebras, NY-London, 1961.

[11] Kostant B., Lie algebra cohomology and the generalized Borel-Weil theorem, Ann. Math. 74, 1961, 329-387

[12] Leger G., Luks E, Cohomology of nilradicals of Borel subalgebras, Trans. Amer. Math. Soc. 195, 1974, 305-316

[13] Saletan E., Contraction of Lie groups, J. Math. Phys. 2, 1961, 1-22 
[14] Tolpygo A., The cohomology of parabolic Lia algebras, Mat. Zametki 12, 1972, 251-255 English translation in Math. Notes 12 (1972), 585-587 (1973)

[15] Tolpygo A., Cohomology of nilpotent Lie algebras and its generating functions, Uspehi Mat. Nauk, 34, 1979, n.1, 245-246 English translation: Russian Math. Surveys 34 (1979), no. 1, 243-244

[16] Tolpygo A. K., Cohomology of Lie algebras and norms of polynomials, (Russian) In: Onishchik A. (ed.) Problems in group theory and homological algebra, Yaroslav. Gos. Univ., Yaroslavl, 1981, 28-38

[17] Tolpygo A. K., Newton polyhedra and the inverse Borel-Weil theorem, Funktsional. Anal. i Prilozhen. 17, 1983, no. 3, 55-60 English translation: Functional Anal. Appl. 17 (1983), no. 3, 207-212

[18] Tolpygo A. K., Cohomology of infinite-dimensional Borel algebras. Ukrain. Mat. Zh. 42, 1990, no. 9, 1278-1283 (Russian); translation in Ukrainian Math. J. 42, 1990, no. 9, 1137-1141

[19] Tolpygo A. K., Estimation of the cohomology of Verma modules. Uspekhi Mat. Nauk 48, 1993, no. 1 (289), 185-186 translation in Russian Math. Surveys 48 (1993), no. 1, 193-194

[20] Tolpygo A. K., Lie algebra cohomology and generating functions. In: D. Leites (ed.) Seminar on supermanifolds 29, 1988-11, 34 pp.

[21] Williams F., Laplace operators and the $\mathfrak{b}$-module structure of certain cohomology groups, Trans. Amer. Math. Soc. 197, 1974, 1-57

This article may be accessed via WWW at http://www.rmi.acnet.ge/hha/ or by anonymous ftp at

$$
\text { ftp://ftp.rmi.acnet.ge/pub/hha/volumes/2004/n1a6/v6n1a6.(dvi,ps,pdf) }
$$

\footnotetext{
Alexei Tolpygo

mleites@mathematik.su.se; tolpygo@kcpik.org.ua

Department of Mathematics,

University of Stockholm,

Roslagsv. 101, Kräftriket hus 6, SE-106 91, Stockholm, Sweden;
} 